\title{
LOCAL ORDERS WHOSE LATTICES ARE DIRECT SUMS OF IDEALS
}

\author{
JEREMY HAEFNER
}

\begin{abstract}
Let $R$ be a complete local Dedekind domain with quotient field $K$ and let $\Lambda$ be a local $R$-order in a separable $K$-algebra. This paper classifies those orders $\Lambda$ such that every indecomposable $R$-torsionfree $\Lambda$-module is isomorphic to an ideal of $\Lambda$. These results extend to the noncommutative case some results for commutative rings found jointly by this author and L. Levy.
\end{abstract}

\section{INTRODUCTION}

Let $R$ be a complete local Dedekind domain with quotient field $K$ and $\Lambda$ a module finite $R$-order in a separable, finite-dimensional $K$-algebra. A $\Lambda$ lattice is a finitely-generated, $R$-torsionfree $\Lambda$-module and an order $\Lambda$ is called sigma- $I$ if every $\Lambda$-lattice is isomorphic to a direct sum of ideals of $\Lambda . \Lambda$ is local provided $\Lambda / \operatorname{rad} \Lambda$ is a division ring. This paper deals with the following questions: Which local $R$-orders are sigma- $I$ and what is their structure?

Current interest in sigma- $I$ orders dates back to a ubiquitous paper $[B]$ which in part considers sigma- $I$ rings that are local commutative, Noetherian, 1dimensional, reduced rings with finitely generated integral closure. Nazarova and Roiter [NR] and Greither [G] also have results pertaining to the commutative sigma- $I$ problem. Finally, Haefner and Levy give a classification of the commutative sigma- $I$ rings in [HL].

While a solution seems a bit more elusive for noncommutative orders, there are results which are useful in the characterization of noncommutative local sigma-I orders. For example, a generalization (due to Roiter [R'66]) of a theorem of Bass states that every order with the 2-generator property (i.e., every left or right ideal can be generated by two elements) is sigma- $I$. In addition to the 2-generator condition, there are two other properties closely related to sigma- $I$. An order $\Lambda$ is Gorenstein if $\left({ }_{\Lambda} \Lambda\right)^{*}=\operatorname{Hom}_{R}(\Lambda \Lambda, R)$ is projective as a right $\Lambda$-module; and $\Lambda$ is Bass if every overorder is Gorenstein. Drozd, Kiricenko and Roiter, in a paper [DKR] that completely classifies Bass orders, prove every Bass order is sigma- $I$ and in certain local cases, the converse.

The thrust of this paper is to identify those local sigma- $I$ orders which are not Bass. To do this, we must know when an order has finite representation type

Received by the editors November 16, 1987 and, in revised form, November 10, 1988.

1980 Mathematics Subject Classification (1985 Revision). Primary 16A10, 16A64; Secondary 16A33, 16A48. 
(FRT); that is, when an order has finitely many nonisomorphic indecomposable lattices. Fortunately, primary orders having FRT are classified by Drozd and Kiricenko in [DK'73]. In their case-by-case analysis, they present much information concerning the structure of FRT rings as well as three necessary and sufficient conditions for a certain local order to be sigma- $I$. However they do not determine the structure of all local sigma- $I$ orders.

In addition to the FRT information of [DK'73], we will use certain pullback constructions of the regular modules ${ }_{\Lambda} \Lambda$ and $\Lambda_{\Lambda}$. This pullback perspective provides explicit descriptions of local sigma-I orders, as seen in $\S 3$. It turns out that the noncommutative structure is strikingly analogous to the commutative situation as explored in [HL].

$\S 1$ explains the pullback point of view and contains some definitions, notations and useful lemmas. $\S 2$ provides new characterizations of sigma- $I$ orders. $\S 3$ is devoted to examples and explicit descriptions of sigma- $I$ orders and, finally, the last section contains some questions for further investigation.

Notation. The following notation and terminology will be fixed for the remainder of this paper:

(1) If $X$ is a module over a ring $R$, then $l_{R}(X)$ denotes the composition length of $X$. In particular, $l_{R}(X)=n<\infty$ if $X$ has a finite composition series of length $n$; otherwise, $l_{R}(X)=\infty$.

(2) If $M$ is a right $\Lambda$-lattice (for an $R$-order $\Lambda$ ), then $M$ is uniform if $M \otimes_{R} K$ is a simple right module for the separable $K$-algebra $A=\Lambda \otimes_{R} K$.

(3) For any $R$-order $\Lambda$, let $\tilde{\Lambda}$ denote the intersection of all maximal orders in $A$ containing $\Lambda$. (Note that maximal orders containing $\Lambda$ exist since the algebra $A$ is separable; see [CR].)

\section{THE PULLBACK PERSPECTIVE}

In this section, we study pullbacks of rings and lattices in order to obtain a precise description of the structure of orders. The results of this section are essential for the characterization of sigma- $I$ orders in $\S 2$. Throughout this section, $\Gamma$ denotes an arbitrary ring with 1 .

1.1 Definition. Suppose that $M_{1}, M_{2}, \ldots, M_{n}$ and $\bar{M}$ are rings (or modules over $\Gamma$ ) and that there exist ring (or module) surjections $f_{i}: M \rightarrow \bar{M}$ for all $i$. Define $M=\left\{\left(x_{1}, \ldots, x_{n}\right) \in M_{1} \oplus \cdots \oplus M_{n}: f_{i}\left(x_{i}\right)=f_{j}\left(x_{j}\right)\right.$ for all $1 \leq i, j \leq n\} ; M$ is called the $n$-fold pullback of $M_{1}, M_{2}, \ldots, M_{n}$ by maps $f_{1}, f_{2}, \ldots, f_{n}$ (over $\bar{M}$ ). Pictorially, we have the following commutative diagram:

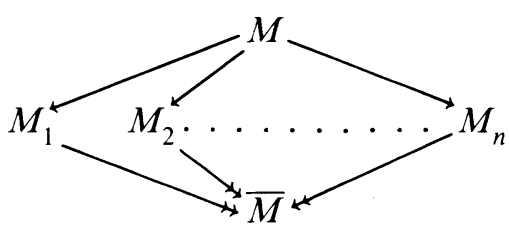


where the top row of arrows are the projections of $M$ onto $M_{i}$. Denote $M$ by

or just

$$
M=\operatorname{pbk}\left(f_{1}, \ldots, f_{n}: M_{1}, \ldots, M_{n} \rightarrow \bar{M}\right)
$$

$$
M=\operatorname{pbk}\left(M_{1}, \ldots, M_{n} \rightarrow \bar{M}\right) .
$$

It is clear that $M$ is a ring (module) if the maps are ring (module) surjections. We denote 2 -fold pullbacks by

$$
M=\operatorname{pbk}\left(f_{1}: M_{1} \rightarrow \bar{M} \nleftarrow M_{2}: f_{2}\right)
$$

or just

$$
M=\operatorname{pbk}\left(M_{1} \rightarrow \bar{M} \leftarrow M_{2}\right) .
$$

1.2 Remark. Every $n$-fold pullback

$$
M=\operatorname{pbk}\left(f_{1}, \ldots, f_{n}: M_{1}, \ldots, M_{n} \rightarrow \bar{M}\right)
$$

is a subdirect sum of $M_{1}, \ldots, M_{n}$. To see this, define $\pi_{i}: M \rightarrow M_{i}$ as the projection of $M$ into $M_{i}$; observe that the $\pi_{i}$ are surjections since the $f_{i}$ are surjections.

Lemma 1.3 proves a partial converse to the above remark; that is, any subdirect sum of $M_{1}$ and $M_{2}$ is a 2-fold pullback. We note in Remark 1.4, however, that an arbitrary subdirect sum of $M_{1}, \ldots, M_{n}$ need not be an $n$ fold pullback.

1.3 Lemma. Suppose $M, M_{1}, M_{2}, N, N_{1}, \bar{N}$ are rings (or modules over a ring $\Gamma)$ such that $M$ is a subdirect sum of $M_{1}$ and $M_{2}$. Let $\pi_{1}$ and $\pi_{2}$ be the projections from $M$ onto $M_{1}$ and $M_{2}$, respectively. Set

$$
\bar{M}=M /\left(\operatorname{ker} \pi_{1}+\operatorname{ker} \pi_{2}\right) .
$$

For $i=1,2$, define $f_{i}: M_{i} \rightarrow \bar{M}$ to be the extension to $M_{i}$ of the canonical map $\eta$ from $M$ to $\bar{M}$ (since $\left.\operatorname{ker} \pi_{i} \subset \operatorname{ker} \eta\right)$. Then:

(1) $M=\operatorname{pbk}\left(f_{1}: M_{1} \rightarrow \bar{M} \nleftarrow M_{2}: f_{2}\right)$.

(2) $\operatorname{ker} f_{1}$ is the largest $\Gamma$-submodule of $M_{1}$ such that $\left(\operatorname{ker} f_{1}\right) \oplus 0 \subset M \subset$ $M_{1} \oplus M_{2}$. Similarly, $\operatorname{ker} f_{2}$ is the largest $\Gamma$-submodule of $M_{2}$ such that $0 \oplus$ $\left(\operatorname{ker} f_{2}\right) \subset M \subset M_{1} \oplus M_{2}$.

(3) If $M_{i}$ is identified with its "zero section" in $M_{1} \oplus M_{2}$ (e.g. $\left.M_{1} \equiv M_{1} \oplus 0\right)$, then $\operatorname{ker} f_{i}=M \cap M_{i}$.

(4) $\operatorname{ker} \pi_{1}=0 \oplus \operatorname{ker} f_{2}$ and $\operatorname{ker} \pi_{2}=\operatorname{ker} f_{1} \oplus 0$ so, in particular, $\operatorname{ker} \pi_{1}+$ $\operatorname{ker} \pi_{2}=\operatorname{ker} f_{1} \oplus \operatorname{ker} f_{2}$.

(5) The Inclusion Property holds: Suppose $N_{1}$ contains $M_{1}$ and

$$
N=\operatorname{pbk}\left(g_{1}: N_{1} \rightarrow \bar{N} \leftarrow M_{2}: g_{2}\right)
$$

for some epimorphisms $g_{1}$ and $g_{2}$. Then $M \subset N$ if and only if there is an epimorphism $\alpha: \bar{M} \rightarrow \bar{N}$ such that the following diagram commutes:

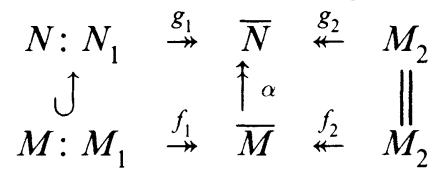


Proof. (1), (5) These results can be obtained by a slight modification of the proof in [HL, §2].

(2) If ker $f_{1} \subset X$ are $\Gamma$-submodules of $M_{1}$ such that $X \oplus 0 \subset M$, then $f_{1}(X)=0$ since $f_{2}(0)=0$. Hence $X=\operatorname{ker} f_{1}$.

(3) Since $M \cap M_{1}=M \cap\left(M_{1} \oplus 0\right) \subset M$, then $f_{1}\left(M \cap M_{1}\right)=0$ and so $M \cap M_{1} \subset \operatorname{ker} f_{1}$. But it is clear that $\operatorname{ker} f_{1} \subset M \cap M_{1}$ and so $\operatorname{ker} f_{1}=M \cap M_{1}$.

(4) From the Diamond Lemma 2.3 of [HL], $\operatorname{ker} \pi_{1}+\operatorname{ker} \pi_{2}=\operatorname{ker} \eta \supset \operatorname{ker} f_{1} \oplus$ $\operatorname{ker} f_{2}$. For the opposite inclusion, project $\operatorname{ker} \eta$ into $M_{1}$ and $M_{2}$. By the pullback structure, these projections are contained in $\operatorname{ker} f_{i}$, respectively.

The next remark shows the connection between $n$-fold and 2 -fold pullbacks.

1.4 Remarks. (1) Every subdirect sum of $M_{1}, \ldots, M_{n}$ can be described as a 2-fold pullback recursively.

The proof of this fact is by induction on $n$. The case $n=2$ is just an application of Lemma 1.3. For $n>2$, let $M$ be a subdirect sum of $M_{1}, \ldots, M_{n}$ where each $M_{i}$ is a module over some fixed ring $\Gamma$. Let $\pi_{i}: M \rightarrow M_{i}$ be the projections of $M$ onto $M_{i}$. Let $W$ be the projection of $M$ into the module $M_{1} \oplus \cdots \oplus M_{n-1}$; that is, $W=\left\{\left(m_{1}, \ldots, m_{n-1}\right):\right.$ there is some $m_{n} \in M_{n}$ such that $\left.\left(m_{1}, \ldots, m_{n}\right) \in M\right\}$. It is easy to see that $M$ is a subdirect sum of $W$ and $M_{n}$. By Lemma 1.3, $M$ is a 2-fold pullback:

$$
M=\operatorname{pbk}\left(h: W \rightarrow \bar{M} \nleftarrow M_{n}: f_{n}\right)
$$

for some epimorphism $h: W \rightarrow \bar{M}$. This completes the proof.

(2) Every $n$-fold pullback, as in Definition 1.1, can also be described as a 2-fold pullback. This follows from Remark 1.2 and Remark 1.4(1) above.

As 3-fold pullbacks play a significant role in what follows, we point out that in particular any 3-fold pullback $M=\operatorname{pbk}\left(f_{1}, f_{2}, f_{3}: M_{1}, M_{2}, M_{3} \rightarrow \bar{M}\right)$ can be identified with

$$
N=\operatorname{pbk}\left(f: W \rightarrow \bar{M} \leftarrow M_{3}: f_{3}\right),
$$

where $W=\operatorname{pbk}\left(f_{1}: M_{1} \rightarrow \bar{M} \nleftarrow M_{2}: f_{2}\right)$ and $f$ is the induced homomorphism from $W$ onto $\bar{M}$ (namely, $f\left(m_{1}, m_{2}\right)=f_{1}\left(m_{1}\right)=f_{2}\left(m_{2}\right)$ ).

(3) A subdirect sum $M$ of $M_{1}, \ldots, M_{n}$ need not be an $n$-fold pullback (although it is a 2-fold pullback by (1)). For example, if $\Lambda=\mathbb{Z}_{2}[X] /\left(1-X^{4}\right)$ (where, as usual, $\mathbb{Z}_{2}$ denotes the integers localized at prime 2 ), then $\Lambda$ is a subdirect sum of $\mathbb{Z}_{2}[X] /(1-X), \mathbb{Z}_{2}[X] /(1+X)$ and $\mathbb{Z}_{2}[X] /\left(1+X^{2}\right)$. It turns out that $\Lambda$ is a 2 -fold pullback of $\mathbb{Z}_{2}[X] /\left(1-X^{2}\right)$ and $\mathbb{Z}_{2}[X] /\left(1+X^{2}\right)$ but it is not the 3-fold pullback of the three rings above. This is the fundamental difference between "triad" and "special quasi-triad" defined in $\S 2$.

The inclusion property is useful for working with pullbacks; so is the following variant. 
1.5 Corollary. Let $M, N, O$ be $\Gamma$-modules with pullback structures

$$
\begin{aligned}
M & =\operatorname{pbk}\left(m_{1}: M_{1} \rightarrow \bar{M} \leftarrow M_{2}: m_{2}\right), \\
N & =\operatorname{pbk}\left(n_{1}: N_{1} \rightarrow \bar{N} \leftarrow M_{2}: n_{2}\right), \\
O & =\operatorname{pbk}\left(o_{1}: O_{1} \rightarrow \bar{O} \leftarrow M_{2}: o_{2}\right),
\end{aligned}
$$

where $M_{1} \subset N_{1} \subset O_{1}, M \subset N \cap O$ and $\bar{M}$ is an Artinian, uniserial module such that $l_{\Gamma}(\bar{M})=m$. Then:

(1) $\bar{N}$ and $\bar{O}$ are Artinian and uniserial, each with length $\leq m$.

(2) $N \subset O$ if and only if $l_{\Gamma}(\bar{N}) \geq l_{\Gamma}(\bar{O})$.

Proof. (1) Apply Lemma 1.3 above.

(2) If $N \subset O$ then, by (4) of Lemma 1.3, there exists an epimorphism $\alpha: \bar{N} \rightarrow \bar{O}$ and so $l_{\Gamma}(\bar{N}) \geq l_{\Gamma}(\bar{O})$.

Conversely, by 1.3 , it suffices to show that there exists an epimorphism $\gamma: \bar{N} \rightarrow \bar{O}$ making the suitable diagram commute. But again by 1.3 , there exists epimorphisms $\alpha: \bar{M} \rightarrow \bar{N}$ and $\beta: \bar{M} \rightarrow \bar{O}$. Since $\bar{M}$ is uniserial and $l_{\Gamma}(\bar{N}) \geq l_{\Gamma}(\bar{O})$, then $\alpha$ factors through $\beta$; that is, there exists $\gamma: \bar{N} \rightarrow \bar{O}$ such that $\gamma \alpha=\beta$.

We know when one pullback is contained in another by Corollary 1.5. We show in the last result of this section how to construct a larger pullback from a smaller one.

1.6 Corollary. Let $M, M_{1}, M_{2}$ and $\bar{M}$ be as in Corollary 1.5. Suppose $\bar{N}$ is a $\Gamma$-module and $\alpha: \bar{M} \rightarrow \bar{N}$ is a $\Gamma$-epimorphism. Set $n_{i}=\alpha \circ m_{i}$. Then the $\Gamma$-module

$$
N=\operatorname{pbk}\left(n_{1}: M_{1} \rightarrow \bar{N} \leftarrow M_{2}: n_{2}\right)
$$

contains $M$.

Proof. This follows from Corollary 1.5. An alternative proof is via the inclusion property of 1.3 .

\section{Characterizations OF LOCAL SIGMA-I ORDERS}

In [HL], a commutative local sigma- $I$ order (with suitable hypotheses) was shown to be either (a) a Bass ring, or (b) a "triad" of three discrete rank one valuation rings pulled back over a field, or (c) a slightly more complicated subdirect sum of three discrete rank one valuation rings, called a "special quasi-triad".

In this section, we give new definitions in the noncommutative setting for "triad" and "special quasi-triad", using very easily stated properties. We justify the terminology by proving equivalences that show these definitions generalize the commutative terminology. Our goal is the following result, which is the main theorem of this article.

2.1 Theorem (Classification of local sigma-I orders). Let $R$ be a complete local Dedekind domain with quotient field $K$ and let $\Lambda$ be a local $R$-order in a 
separable $K$-algebra $A$. Then $\Lambda$ is sigma-I if and only if $\Lambda$ is either

(i) a Bass order, or

(ii) a triad, or

(iii) a special quasi-triad.

The definitions of "triad" and "special quasi-triad" for this more general setting are given in 2.2. We delay the proof of Theorem 2.1 until we have developed enough machinery. Throughout $R, K, \Lambda$ and $A$ will be as in the hypothesis of Theorem 2.1 .

2.2 Definitions. Suppose $\Lambda$ has finite representation type (FRT) and $l_{A}(A)=$ 3. Then $\Lambda$ is called a triad provided $\operatorname{rad} \Lambda=\operatorname{rad} \tilde{\Lambda} ; \Lambda$ is called a special quasitriad provided $\Lambda$ has a unique minimal overmodule (in $A$ ), $T=\Lambda+\operatorname{rad} \tilde{\Lambda}$.

The above definitions were easily stated, but their utility is hindered because they lack structure and detail. Furthermore, the triad definition is seemingly incompatible with Bass' definition as a 3-fold pullback of three discrete valuation rings [B]. All such worries are resolved by Theorems 2.6 and 2.12 where we give the structure of triads and special quasi-triads in terms of the pullback machinery.

The following proposition summarizes some relevant results of Drozd, Kiricenko and Roiter.

2.3 Proposition. Given $R, K, \Lambda$ and $A$ as in the hypothesis of 2.1 .

(1) [R'68] If $\Lambda$ is sigma-I, then $\Lambda$ has $F R T$.

(2) [DK'73, p. 717] If $\Lambda$ has FRT, then $l_{A}(A) \leq 3$.

(3) [DKR, 12.1] For $l_{A}(A)<3, \Lambda$ is sigma-I if and only if $\Lambda$ is Bass.

(4) [DKR, 10.3; DK'72, 3.3] If $\Lambda$ is Gorenstein but not Bass, then $\Lambda$ is contained in a unique minimal overring $O$ (in $A$ ), and, furthermore, $O$ is not Gorenstein.

(5) [DK'73, 1.2] The order $\Lambda$ has FRT if and only if all of the following three conditions hold:

(a) $\tilde{\Lambda}$ is hereditary,

(b) $\tilde{\Lambda} / \Lambda$ is generated by two elements, and

(c) $\operatorname{rad} \tilde{\Lambda} / \Lambda$ is a cyclic $\Lambda$-module.

(6) [DK'73, p. 717] If $\Lambda$ has FRT and $l_{A}(A)=3$, then

(a) every uniform $\Lambda$-lattice is also a (uniform) $\tilde{\Lambda}$-lattice;

(b) every uniform $\Lambda$-lattice is uniserial and cyclic;

(c) there exist three uniform right $\Lambda$ (and so $\tilde{\Lambda}$ ) lattices $X_{1}, X_{2}$ and $X_{3}$ such that $\tilde{\Lambda}=X_{1} \oplus X_{2} \oplus X_{3}$; and

(d) every uniform $\Lambda$ (and so $\tilde{\Lambda}$ ) lattice $M$ is isomorphic to one of $X_{1}, X_{2}$, $X_{3}$.

(7) The statement of (6) holds if "right" is replaced by "left". 
2.4 Right module notation. Suppose $\Lambda$ has FRT and $l_{A}(A)=3$. Let $X_{1}, X_{2}$, $X_{3}$ be the uniform right modules given by 2.3(6). For each $i$, put $Y_{i}=$ $\operatorname{rad} X_{i}$, which is maximal in $X_{i}$ by 2.3(6). Let $\left\{e_{1}, e_{2}, e_{3}\right\}$ be a complete set of orthogonal primitive idempotents of $A$; note that $e_{i} \cdot \widetilde{\Lambda}=X_{i}$. By 2.3(6)(a), it is easy to see that $e_{i} \cdot \widetilde{\Lambda}=X_{i}=e_{i} \cdot \Lambda$; let $\pi_{i}$ be the projection of $\Lambda$ onto $X_{i}$ (via left multiplication by $e_{i}$ ). Set

$$
\Gamma=\left(e_{1}+e_{2}\right) \Lambda, \quad \bar{\Gamma}=\frac{\Gamma}{\left(\Gamma \cap X_{1}\right)+\left(\Gamma \cap X_{2}\right)} \quad \text { and } \quad \bar{\Lambda}=\frac{\Lambda}{\left(\Lambda \cap X_{3}\right)+(\Lambda \cap \Gamma)} .
$$

(We identify $X_{i}$ with its zero section in $\tilde{\Lambda}_{\tilde{\Lambda}}=X_{1} \oplus X_{2} \oplus X_{3}$, as we will throughout this paper.) Set $\pi$ to be the projection of $\Lambda$ onto $\Gamma$, and note that $\Gamma \subset X_{1} \oplus X_{2}$. Also let $\eta_{\Gamma}\left(\eta_{\Lambda}\right.$, respectively) be the canonical map from $\Gamma$ onto $\bar{\Gamma}$ (from $\Lambda$ onto $\bar{\Lambda}$, respectively).

It is straightforward to check that $\operatorname{ker} \pi=\operatorname{ker} \pi_{1} \cap \operatorname{ker} \pi_{2}=\Lambda \cap X_{3}$, so the map $\pi_{i}$ can be factored through $\Gamma$. In other words, we have the following commutative diagrams for $i=1,2$ :

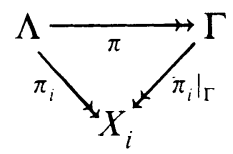

For notational convenience, we use $\left.\pi_{i}\right|_{\Gamma}$ to denote the projection from $\Gamma$ onto $X_{i}$.

Now ker $\left.\pi_{1}\right|_{\Gamma}=\Gamma \cap X_{2}$ and $\left.\operatorname{ker} \pi_{2}\right|_{\Gamma}=\Gamma \cap X_{1}$. As a result, for $i=1,2$, the map $\eta_{\Gamma}: \Gamma \rightarrow \bar{\Gamma}$ factors through $X_{i}$ by a map $f_{i}: X_{i} \rightarrow \bar{\Gamma}$; that is, we have the following diagrams commute for $i=1,2$ :

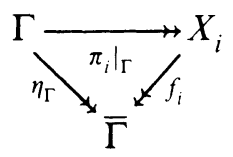

Similarly, $\operatorname{ker} \pi=\operatorname{ker} \pi_{1} \cap \operatorname{ker} \pi_{2}=\Lambda \cap X_{3}$ and $\operatorname{ker} \pi_{3}=\Lambda \cap(\Gamma \oplus 0)$. Again, the canonical map $\eta_{\Lambda}: \Lambda \rightarrow \bar{\Lambda}$ factors through both $\Gamma$ and $X_{3}$; that is, there are maps $f: \Gamma \rightarrow \bar{\Lambda}$ and $f_{3}: X_{3} \rightarrow \bar{\Lambda}$ making these diagrams commute:

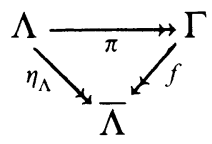

and

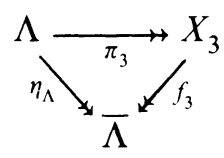


Pictorially, we have:

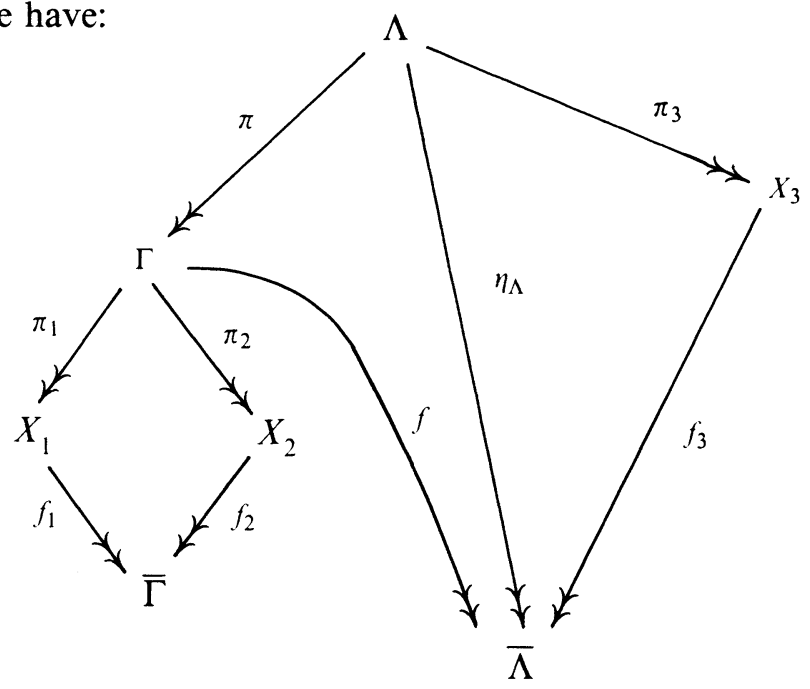

Finally, define $P_{i}=\left(X_{j} \oplus X_{k}\right) \cap \Lambda$, where $j, k \in\{1,2,3\}-\{i\}$ for each $i=1,2,3$. Note that $P_{i}=\operatorname{ker} \pi_{i}$ and $P_{1} \cap P_{2}=\operatorname{ker} \pi$.

The picture above suggests that $\Lambda$ and $\Gamma$ are pullbacks. Indeed, this is the case, as seen next.

\subsection{Proposition. Assume Notation 2.4 holds.}

(1) $\Lambda_{\Lambda}$ is a subdirect sum of $\tilde{\Lambda}_{\tilde{\Lambda}}=X_{1} \oplus X_{2} \oplus X_{3}$ and $\operatorname{rad} \Lambda \subset \operatorname{rad} \tilde{\Lambda}=$ $Y_{1} \oplus Y_{2} \oplus Y_{3}$.

(2) $\Gamma_{\Lambda}=\operatorname{pbk}\left(f_{1}: X_{1} \rightarrow \bar{\Gamma} \nleftarrow X_{2}: f_{2}\right)$.

(3) $\Lambda_{\Lambda}=\operatorname{pbk}\left(f: \Gamma \rightarrow \bar{\Lambda} \nleftarrow X_{3}: f_{3}\right)$.

(4) $\Gamma \cong \Lambda /\left(P_{1} \cap P_{2}\right), \bar{\Gamma} \cong \Lambda /\left(P_{1}+P_{2}\right)$ and $\bar{\Lambda} \cong \Lambda /\left(P_{1} \cap P_{2}+P_{3}\right)$.

(5) $\Gamma$ has a unique maximal $\Lambda$-submodule, namely $\left(e_{1}+e_{2}\right) \cdot \operatorname{rad} \Lambda$.

(6) $\bar{\Gamma}$ and $\bar{\Lambda}$ are each Artinian, uniserial modules.

(7) The left regular module ${ }_{\Lambda} \Lambda$ has a structure analogous to that for $\Lambda_{\Lambda}$ as described in (1)-(4).

Proof. (1) Since the $e_{i}$ are the primitive idempotents of $A$, then $e_{i} \cdot \Lambda \subset e_{i} \cdot \tilde{\Lambda}$ are uniform $\Lambda$-modules. But every $e_{i} \cdot \Lambda$ is also a $\tilde{\Lambda}$-module by 2.3(6)(a) and so $e_{i} \cdot \Lambda=e_{i} \cdot \widetilde{\Lambda}=X_{i}$. Thus, $\Lambda$ is a subdirect sum as desired.

Since $e_{i} \cdot \Lambda=X_{i}$, we have either $e_{i} \cdot \operatorname{rad} \Lambda=Y_{i}$ or $\operatorname{rad} \Lambda \subset Y_{1} \oplus Y_{2} \oplus Y_{3}$. But $Y_{1} \oplus X_{2} \oplus X_{3}, X_{1} \oplus Y_{2} \oplus X_{3}$ and $X_{1} \oplus X_{2} \oplus Y_{3}$ are maximal right ideals of $\tilde{\Lambda}$, and so $\operatorname{rad} \tilde{\Lambda} \subset Y_{1} \oplus Y_{2} \oplus Y_{3}$. On the other hand,

$$
\frac{\tilde{\Lambda}}{Y_{1} \oplus Y_{2} \oplus Y_{3}} \cong \frac{X_{1}}{Y_{1}} \oplus \frac{X_{2}}{Y_{2}} \oplus \frac{X_{3}}{Y_{3}}
$$

is obviously semisimple; hence $\operatorname{rad} \tilde{\Lambda}=Y_{1} \oplus Y_{2} \oplus Y_{3}$. 
(2), (3) It follows from (1) that $\Gamma_{\Lambda}$ is a subdirect sum of $X_{1}$ and $X_{2}$, and $\Lambda_{\Lambda}$ is a subdirect sum of $\Gamma$ and $X_{3}$. Thus, by 1.3(1), it suffices to show

$$
\bar{\Gamma} \cong \frac{\Gamma}{\operatorname{ker}\left(\Gamma \rightarrow X_{1}\right)+\operatorname{ker}\left(\Gamma \rightarrow X_{2}\right)}=\frac{\Gamma}{\operatorname{ker}\left(\left.\pi_{1}\right|_{\Gamma}\right)+\operatorname{ker}\left(\left.\pi_{2}\right|_{\Gamma}\right)}
$$

and the analogous statements for $\bar{\Lambda}$. It is not difficult to see that $\operatorname{ker}\left(\left.\pi_{1}\right|_{\Gamma}\right)=$ $\Gamma \cap X_{2}$ and $\operatorname{ker}\left(\left.\pi_{2}\right|_{\Gamma}\right)=\Gamma \cap X_{1}$. Consequently, using the definition of $\bar{\Gamma}$ given in Notation 2.4, $\bar{\Gamma}$ is as desired. In a similar manner, we obtain the results for $\bar{\Lambda}$.

(4) These follow from Notation 2.4.

(5) This is clear since $\Gamma=\left(e_{1}+e_{2}\right) \cdot \Lambda$ and $\Lambda$ is local.

(6) This follows since $X_{3}$ is uniserial.

(7) By symmetric arguments used in the proofs of (1) through (4).

Using the pullback machinery, we now give a pragmatic description of the structure of triads.

2.6 Theorem (Characterizations of triads). Assume $R, \Lambda, K, A$ are as in the hypotheses of Theorem 2.1 and set $U=\Lambda / \operatorname{rad} \Lambda$. Then the following statements are equivalent:

(1) $\Lambda$ is a triad.

(2) $\Lambda$ has FRT and $\Lambda$ is a 3-fold pullback of uniform right $\Lambda$-lattices $X_{1}, X_{2}$, $X_{3}$ by right $\Lambda$-epimorphisms $t_{i}: X_{i} \rightarrow U$ (over $\left.U\right)$; that is, $\Lambda=\left\{\left(x_{1}, x_{2}, x_{3}\right) \in\right.$ $\left.X_{1} \oplus X_{2} \oplus X_{3}: t_{1}\left(x_{1}\right)=t_{2}\left(x_{2}\right)=t_{3}\left(x_{3}\right)\right\}$.

(3) $\Lambda$ has FRT and $\Lambda$ is a 3-fold pullback of uniform left $\Lambda$-lattices $\mathfrak{X}_{1}, \mathfrak{X}_{2}$, $\mathfrak{X}_{3}$ by left $\Lambda$-epimorphisms $s_{i}: \mathfrak{X}_{i} \rightarrow U$ (over $U$ ); that is, $\Lambda \cong\left\{\left(x_{1}, x_{2}, x_{3}\right) \in\right.$ $\left.\mathfrak{X}_{1} \oplus \mathfrak{X}_{2} \oplus \mathfrak{X}_{3}: s_{1}\left(x_{1}\right)=s_{2}\left(x_{2}\right)=s_{3}\left(x_{3}\right)\right\}$.

Proof. (1) $\Rightarrow$ (2) Since $\Lambda$ is a triad, $\Lambda$ has FRT, $l_{A}(A)=3$ and $\operatorname{rad} \Lambda=$ $\operatorname{rad} \tilde{\Lambda}$. Thus, using the notation of 2.4 and $2.5, \operatorname{rad} \Lambda=Y_{1} \oplus Y_{2} \oplus Y_{3}$ and $\Lambda_{\Lambda}$ is a subdirect sum of $X_{1} \oplus X_{2} \oplus X_{3}=\tilde{\Lambda}_{\tilde{\Lambda}}$.

We first show that $\operatorname{ker} f_{i}=Y_{i}$. As described in 1.3, $\operatorname{ker} f_{i}=\Lambda \cap X_{i}$. It suffices to show $\Lambda \cap X_{3}=Y_{3}$ as the other cases are analogous. Clearly, $Y_{3} \subset \Lambda \cap X_{3}$ since $\operatorname{rad} \Lambda=Y_{1} \oplus Y_{2} \oplus Y_{3}$. If $\Lambda \cap X_{3}=X_{3}$, then the inclusions

$$
\operatorname{rad} \Lambda=Y_{1} \oplus Y_{2} \oplus Y_{3} \subset Y_{1} \oplus Y_{2} \oplus X_{3}
$$

are proper, a contradiction to $\Lambda$ local. By a similar fashion, it follows that $\Gamma \cap X_{i}=Y_{i}$ for $i=1,2$ and $\Lambda \cap \Gamma=Y_{1} \oplus Y_{2}$.

In particular,

$$
\bar{\Gamma}=\frac{\Gamma}{\left(\Gamma \cap X_{1}\right)+\left(\Gamma \cap X_{2}\right)}=\frac{\Gamma}{Y_{1} \oplus Y_{2}}
$$

and

$$
\bar{\Lambda}=\frac{\Lambda}{\left(\Lambda \cap X_{3}\right)+(\Lambda \cap \Gamma)}=\frac{\Lambda}{Y_{1} \oplus Y_{2} \oplus Y_{3}} ;
$$

furthermore, both are simple $\Lambda$-modules. 
Now fix an isomorphism $\psi: \bar{\Lambda} \rightarrow U$ and, respectively, define the maps $t$ and $t_{3}$ as the compositions

$$
t=\psi \circ f: \Gamma \rightarrow U \text { and } t_{3}=\psi \circ f_{3}: X_{3} \rightarrow U .
$$

In particular, we have $\Lambda=\operatorname{pbk}\left(t: \Gamma \rightarrow U \nleftarrow X_{3}: t_{3}\right)$. Now $\operatorname{ker} t=Y_{1} \oplus Y_{2}$ so $t$ can be factored by $\eta_{\Gamma}$, the canonical map from $\Gamma$ onto $\bar{\Gamma}$; i.e., we have the following commutative diagram:

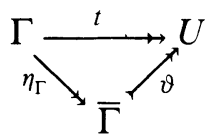

where $\vartheta: \bar{\Gamma} \rightarrow U$ is a $\Lambda$-isomorphism. But, from the definition of $f_{1}$ and $f_{2}$, $\eta_{\Gamma}$ equals the compositions

$$
\Gamma \stackrel{\pi_{1}}{\rightarrow} X_{1} \stackrel{f_{1}}{\rightarrow} \bar{\Gamma} \text { and } \Gamma \stackrel{\pi_{2}}{\rightarrow} X_{2} \stackrel{f_{2}}{\rightarrow} \bar{\Gamma} .
$$

Subsequently, we have the following commutative diagram:

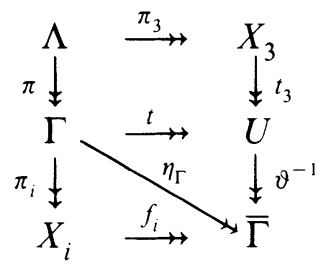

Now set $t_{1}=\vartheta \circ f_{1}$ and $t_{2}=\vartheta \circ f_{2}$ so that

$$
\Gamma=\operatorname{pbk}\left(t_{1}: X_{1} \rightarrow U \leftarrow X_{2}: t_{2}\right) .
$$

As a result, $\left(x_{1}, x_{2}, x_{3}\right) \in \Lambda \Leftrightarrow t\left(x_{1}, x_{2}\right)=t_{3}\left(x_{3}\right) \Leftrightarrow t_{1}\left(x_{1}\right)=t_{2}\left(x_{2}\right)=t\left(x_{1}, x_{2}\right)$ $=t_{3}\left(x_{3}\right)$. Hence, $\Lambda$ is the desired 3 -fold pullback.

(2) $\Rightarrow$ (1) If $\Lambda$ has the form of (2), then $\Lambda$ is contained in a direct sum of three uniform lattices. Consequently, $\Lambda$ has FRT and $l_{A}(A)=3$. Thus, we can use Notation 2.4 to describe $\Lambda$. It suffices to show that $\operatorname{rad} \tilde{\Lambda}=Y_{1} \oplus Y_{2} \oplus Y_{3}$ is the maximal right ideal of $\Lambda$. Clearly,

$$
Y_{1} \oplus Y_{2} \oplus Y_{3} \subset \Lambda
$$

since $Y_{i}=\operatorname{ker}\left(f_{i}: X_{i} \rightarrow U\right)$. Yet $\operatorname{rad} \Lambda \subset \operatorname{rad} \tilde{\Lambda}$ by $2.5(1)$, and so we have $\operatorname{rad} \Lambda \subset \operatorname{rad} \tilde{\Lambda}=Y_{1} \oplus Y_{2} \oplus Y_{3} \subset \operatorname{rad} \Lambda$, as desired.

(1) $\Leftrightarrow$ (3) These proofs are symmetric to those above.

2.7 Remark. We note from the above proof that the triad also has the pullback structure $\Lambda=\operatorname{pbk}\left(t: \Gamma \rightarrow U \leftarrow X_{3}: t_{3}\right)$, where $\Gamma=\operatorname{pbk}\left(t_{1}: X_{1} \rightarrow U \nleftarrow X_{2}: t_{2}\right)$ and $t\left(x_{1}, x_{2}\right)=t_{1}\left(x_{1}\right)=t_{2}\left(x_{2}\right)$. In particular, note that $\operatorname{ker} t_{i}=Y_{i}$ and $\operatorname{ker} t=Y_{1} \oplus Y_{2}$.

There is an analogous theorem (Theorem 2.12) for special quasi-triads but we will first need some information regarding the subrings of a triad. 
2.8 Theorem. Let $\Lambda$ have FRT and $l_{A}(A)=3$ and set $T=\Lambda+\operatorname{rad} \tilde{\Lambda}$. Then

(1) $\Lambda$ is Gorenstein if and only if $\Lambda$ has a unique minimal overmodule, which is also a local non-Gorenstein overorder.

(2) $T$ is a triad such that $\Lambda \subset T \subset \widetilde{\Lambda}, \operatorname{rad} T=\operatorname{rad} \tilde{\Lambda}$ and $T / \operatorname{rad} T \cong$ $\Lambda / \operatorname{rad} \Lambda$ as $\Lambda$-modules.

(3) If $\Lambda$ is a triad, then $\Lambda$ is not Gorenstein. If $\Lambda$ is a special quasi-triad, then $\Lambda$ is Gorenstein.

(4) If $\Lambda$ is a special quasi-triad, then an indecomposable $\Lambda$-lattice is either isomorphic to $\Lambda$ or else is a T-lattice. In particular, a special quasi-triad $\Lambda$ has FRT.

Proof. (1) $(\Rightarrow)$ By [DKR, 10.3; DK'72, 3.7], there exists a unique minimal overorder $O$ which is local. If $O$ is Gorenstein, then, by [DK'72, 3.3], $\Lambda$ would be a local Bass ring. This contradicts $l_{A}(A)=3$ [DKR, 12.1]; hence, $O$ is not Gorenstein.

$(\Leftrightarrow)$ From [DK'72, 2.8] and the fact that $\Lambda$ is local, $\Lambda$ must be injective on the category of $\Lambda$-lattices. Hence, $\Lambda$ is Gorenstein.

(2) Since $\operatorname{rad} \tilde{\Lambda}$ is a 2 -sided ideal of $\widetilde{\Lambda}$, observe that $T$ is a ring with identity such that $\Lambda \subset T \subset \widetilde{\Lambda}$. Set $J=\operatorname{rad} \tilde{\Lambda}$.

Claim. $T$ has no nontrivial idempotents.

Let $e \in T$ be a nonzero idempotent. Write $e=\lambda+y$ where $\lambda \in \Lambda$ and $y \in J$. Now $J$ has no nonzero idempotents: if $x \in J$ such that $x=x^{2}$, then $1-x$ is a unit of $\tilde{\Lambda}$, a contradiction. Since $\Lambda$ is local with $\operatorname{rad} \Lambda \subset J$ then $\lambda \notin \operatorname{rad} \Lambda$ (otherwise $e \in J$ ). So $\lambda$ is a unit of $\Lambda$ and hence of $\tilde{\Lambda}$.

Now $e=e^{2}$ so modulo $J, \lambda \equiv \lambda^{2}$. Since $\lambda$ is a unit, $\lambda \equiv 1 \bmod J$. After renaming, let $e=1+y$ where $y \in J$. But $e=e^{2}$ implies $1+y=1+2 \cdot y+y^{2}$ and so $0=y+y^{2}=y \cdot(1+y)$. Yet $y \in J$ implies $1+y$ is a unit in $\widetilde{\Lambda}$. Thus, $0=y \cdot(1+y) \cdot(1+y)^{-1}=y$ and so $e=1$. This proves the claim.

In particular, $T_{T}$ is indecomposable as a module. But since $R$ is a complete local Dedekind domain, $T$ is semiperfect and so $T \cong \operatorname{End}\left(T_{T}\right)$ is local.

To see that $T$ is indeed the triad, first observe that $\operatorname{rad} \widetilde{\Lambda} \subset \operatorname{rad} T$; consequently,

$$
\frac{T}{\operatorname{rad} \widetilde{\Lambda}}=\frac{\Lambda+\operatorname{rad} \tilde{\Lambda}}{\operatorname{rad} \widetilde{\Lambda}} \cong \frac{\Lambda}{\Lambda \cap \operatorname{rad} \widetilde{\Lambda}}=\frac{\Lambda}{\operatorname{rad} \Lambda}
$$

are simple $\Lambda$ and $T$-modules.

(3) A local Gorenstein ring must have a unique minimal overmodule by (1). However, it is straightforward to see that $\operatorname{pbk}\left(f_{1}: X_{1} \rightarrow U \leftarrow X_{2}: f_{2}\right) \oplus X_{3}$ and $X_{1} \oplus \operatorname{pbk}\left(f_{2}: X_{2} \rightarrow U \leftarrow X_{3}: f_{3}\right)$ are distinct minimal overmodules of the triad by 1.3 and 1.5 .

On the other hand, a special quasi-triad has a unique minimal overmodule, $T=\Lambda+\operatorname{rad} \widetilde{\Lambda}$, which, by (2), is a triad.

(4) This last result stems from the fact that $\Lambda$ is Gorenstein with minimal overorder $T$ (see [CR, 37.13]). 
2.9 Definitions. Let $\Lambda$ be a local order with FRT such that $l_{A}(A)=3$. As before, write $U=\Lambda / \operatorname{rad} \Lambda$ and recall, from 2.3(6), that the submodule structure of any uniform $\Lambda$-lattice is linearly ordered.

(1) The local order $T=\Lambda+\operatorname{rad} \tilde{\Lambda}$ is called the associated triad of $\Lambda$.

(2) A right $\Lambda$-module $M$ will be called a right special quasi-triad module provided there exist a right $\Lambda$-module $\bar{M}$, three uniform right $\Lambda$-lattices $M_{1}$, $M_{2}, M_{3}$ with unique maximal submodules $N_{1}, N_{2}, N_{3}$ respectively and epimorphisms $f_{1}, f_{2}, f_{3}, f$ such that

(a) $M_{\Lambda} \cong \operatorname{pbk}\left(f: \mathscr{M} \rightarrow \bar{M} \nleftarrow M_{3}: f_{3}\right)$, where

$$
\mathscr{M}=\operatorname{pbk}\left(f_{1}: M_{1} \rightarrow \bar{M} \nleftarrow M_{2}: f_{2}\right),
$$

and

(b) there exist epimorphisms $g_{1}$ and $g_{2}$ such that the module $\mathscr{N}=\operatorname{ker} f=$ $\operatorname{pbk}\left(g_{1}: N_{1} \rightarrow U \leftarrow N_{2}: g_{2}\right)$.

Pictorially, we have:

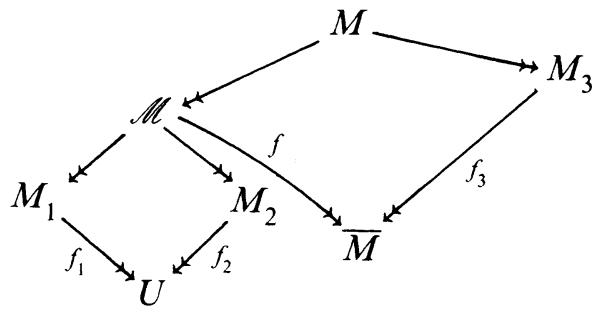

and

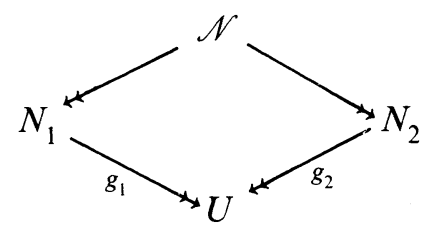

The left special quasi-triad module is analogously defined.

2.10 Remarks. (1) If $O_{i}$ is the unique maximal submodule of $N_{i}$ for $i=1,2$, then it is easy to see that $\operatorname{ker} f_{i}=N_{i}$ and $\operatorname{ker} g_{i}=O_{i}$.

(2) $\mathcal{N}$ is a maximal $\Lambda$-submodule of $N_{1} \oplus N_{2}$ and $N_{1} \oplus N_{2}$ is a maximal $\Lambda$-submodule of $\mathscr{M}$ (by Lemma 1.3).

(3) $\bar{M}=\mathscr{M} / \mathscr{N}$ has composition length 2 .

(4) $\operatorname{ker}\left(f_{3}: M_{3} \rightarrow \bar{M}\right)=O_{3}$ and is the unique largest $\Lambda$-submodule of $N_{3}$.

2.11 Notation. Let $\Lambda$ and $T$ be as in Definition 2.9. We shall keep the notation of 2.4 reserved for $\Lambda$ and shall fix the following notation for $T$ (see Remark 2.7):

$$
T=\operatorname{pbk}\left(t: \Theta \rightarrow U \leftarrow X_{3}: t_{3}\right),
$$

where

$$
\Theta=\operatorname{pbk}\left(t_{1}: X_{1} \rightarrow U \leftarrow X_{2}: t_{2}\right) .
$$


Theorem 2.8 shows that the triad is the "largest" local $R$-order inside $\widetilde{\Lambda}$. We are now able to delve into the structure of the special quasi-triad, again using the pullback perspective.

2.12 Theorem (Characterizations of special quasi-triads). For $R, \Lambda, K, A$ as specified in 2.1, $U=\Lambda / \operatorname{rad} \Lambda$ and $l_{A}(A)=3$, the following statements are equivalent:

(1) $\Lambda$ is a special quasi-triad.

(2) $\Lambda$ is Gorenstein and $\Lambda_{\Lambda}$ is a right special quasi-triad module.

(3) $\Lambda$ is Gorenstein and ${ }_{\Lambda} \Lambda$ is a left special quasi-triad module.

Proof. (1) $\Rightarrow(2)$ By (3) of Theorem 2.8, $\Lambda$ is Gorenstein and $T=\Lambda+\operatorname{rad} \tilde{\Lambda}$ is the associated triad. From (4) of $2.8, \Lambda$ has FRT and $l_{A}(A)=3$; so, using Proposition 2.5, write

$$
\Lambda_{\Lambda}=\operatorname{pbk}\left(f: \Gamma \rightarrow \bar{\Lambda} \leftarrow X_{3}: f_{3}\right),
$$

where

$$
\Gamma_{\Lambda}=\operatorname{pbk}\left(f_{1}: X_{1} \rightarrow \bar{\Gamma} \nleftarrow X_{2}: f_{2}\right) .
$$

If $\bar{\Gamma}=0$, then $\Gamma=X_{1} \oplus X_{2}$. Yet $\Lambda$ has one maximal submodule, and hence so must every homomorphic image of $\Lambda$. Since $\Gamma=X_{1} \oplus X_{2}$ is the projection of $\Lambda$ into $X_{1} \oplus X_{2}$, this is a contradiction. Thus, $\bar{\Gamma} \neq 0$. On the other hand, if $l_{\Lambda}(\bar{\Gamma})>1$, then $\Gamma \oplus X_{3}$ (properly) contains $\Lambda$ (apply the inclusion property of 1.3). But $\Gamma \oplus X_{3}$ does not contain $T$ since the projection of $T$ into $X_{1} \oplus X_{2}$ is $\Theta$ which properly contains $\Gamma$ (apply the inclusion property). This contradicts the uniqueness of $T$ as the minimal overorder of $\Lambda$; hence, $\bar{\Gamma}=U$.

If $l_{\Lambda}(\bar{\Lambda})=0$, then $\Lambda=\Gamma \oplus X_{3}$, a contradiction to $\Lambda$ local. If $l_{\Lambda}(\bar{\Lambda})=1$, then $\bar{\Lambda} \cong U$; it is straightforward to see from Corollary 1.5 and Notation 2.11 that $\Lambda=T$, another contradiction. Thus, $l_{\Lambda}(\bar{\Lambda}) \geq 2$. But if $l_{\Lambda}(\bar{\Lambda})>2$, then, using Corollary 1.6 , we construct the module

$$
M=\operatorname{pbk}\left(h: \Gamma \rightarrow \frac{\bar{\Lambda}}{\operatorname{socle}(\bar{\Lambda})} \leftarrow X_{3}: h_{3}\right)
$$

(since $\bar{\Lambda}$ is uniserial) such that $\Lambda \subset M$. Now $l_{\Lambda}(\bar{\Lambda} / \operatorname{socle}(\bar{\Lambda})) \geq 2$ so by Corollary 1.5, we have $\Lambda \subset M \subset T$ where the inclusions are proper; this contradicts the minimality of $T$ over $\Lambda$, and so $l_{\Lambda}(\bar{\Lambda})=2$.

Let $H=\operatorname{ker}(f: \Gamma \rightarrow \bar{\Lambda})$. To show that $\Lambda_{\Lambda}$ is a special quasi-triad, we must show that $H$ has the form:

$$
H=\operatorname{pbk}\left(g_{1}: Y_{1} \rightarrow \bar{H} \nleftarrow Y_{2}: g_{2}\right),
$$

where $g_{i}$ are some $\Lambda$-epimorphisms. Now $Y_{1} \oplus Y_{2}$ is the unique maximal $\Lambda$-submodule of $\Gamma$ by $2.5(5)$; so either $H \subset Y_{1} \oplus Y_{2}$ or $H=\Gamma$. If $H=\Gamma$, then $l_{\Lambda}(\bar{\Lambda})=l_{\Lambda}(\Gamma / H)=0$, a contradiction. Hence, $H \subset Y_{1} \oplus Y_{2}$.

Next we claim that $H$ is a subdirect sum of $Y_{1}$ and $Y_{2}$. Set $\left.\pi_{1}\right|_{\Gamma}(H)=$ $Z_{1}$ and $\left.\pi_{2}\right|_{\Gamma}(H)=Z_{2}$ so that $H$ is the subdirect sum of $Z_{1}$ and $Z_{2}$. But 
$l_{\Lambda}(\Gamma / H)=2$ so, applying Corollary $1.5, H$ is one of three possibilities: $Y_{1} \oplus Z_{2}$, $Z_{1} \oplus Y_{2}$ or a subdirect sum of $Y_{1}$ and $Y_{2}$, where $Z_{i}$ is the (unique) maximal submodule of $Y_{i}$. Suppose $H=Z_{1} \oplus Y_{2}$. Then because $H=\operatorname{ker} f \supset 0 \oplus Y_{2}$, the map $f: \Gamma \rightarrow \bar{\Lambda}$ factors through $X_{1}$. In other words, there is a map $k: X_{1} \rightarrow \bar{\Lambda}$ such that the following diagram commutes:

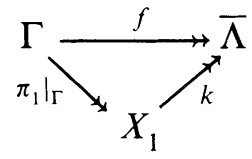

Now construct the $\Lambda$-lattice

$$
M=X_{2} \oplus \operatorname{pbk}\left(k: X_{1} \rightarrow \bar{\Lambda} \nleftarrow X_{3}: f_{3}\right) .
$$

Notice that $\operatorname{ker} k=Z_{1}$ and $\operatorname{ker} f_{3}=Z_{3}$, both of which are maximal in $Y_{i}$. We observe that $\Lambda \subset M$ because of the above commutative diagram. But $T \not \subset M$ because $Y_{1} \oplus 0 \oplus 0 \not \subset M$ (otherwise $Y_{1}=\operatorname{ker} k$, a contradiction). This contradicts the fact that $T$ is the unique minimal overmodule of $\Lambda$. Hence, $H \neq Z_{1} \oplus Y_{2}$. Similarly, $H \neq Y_{1} \oplus Z_{2}$ so $H$ is a subdirect sum of $Y_{1}$ and $Y_{2}$, as claimed.

Consequently, we can use Lemma 1.3 to write

$$
H=\operatorname{pbk}\left(g_{1}: Y_{1} \rightarrow \bar{H} \nleftarrow Y_{2}: g_{2}\right),
$$

where $g_{i}$ are some $\Lambda$-epimorphisms. If $\bar{H}=0$, then $H=Y_{1} \oplus Y_{2}$ and $l_{\Lambda}(\Gamma / H)=1$, a contradiction. Thus, $\bar{H} \neq 0$. If $l_{\Lambda}(\bar{H}) \geq 2$, then by Corollaries 1.5 and 1.6 , we can construct a module

$$
M=\operatorname{pbk}\left(h_{1}: Y_{1} \rightarrow \frac{\bar{H}}{\operatorname{socle}(\bar{H})} \nleftarrow Y_{2}: h_{2}\right)
$$

(since $\bar{H}$ is uniserial) such that $H \subset M \subset Y_{1} \oplus Y_{2} \subset \Gamma$ where the inclusions are proper. This also contradicts $l_{\Lambda}(\Gamma / H)=2$. Thus, $l_{\Lambda}(\bar{H})=1$ and so $\Lambda_{\Lambda}$ is a right special quasi-triad module.

(2) $\Rightarrow$ (3) It suffices to show that ${ }_{\Lambda} \Lambda$ is a left special quasi-triad. Since $\Lambda$ is Gorenstein, ${ }_{\Lambda} \Lambda \cong\left(\Lambda_{\Lambda}\right)^{*}=\operatorname{Hom}_{R}\left(\Lambda_{\Lambda}, R\right)$ and so it suffices to show that $\left(\Lambda_{\Lambda}\right)^{*}$ is a left special quasi-triad. By Definition 2.9 and Remark 2.10(3), $l_{\Lambda}(\Gamma / H)=2$ and $Z_{3}=\operatorname{ker}\left(f_{3}: X_{3} \rightarrow \bar{\Lambda}\right) \subset Y_{3} \subset X_{3}$. Subsequently, there are two short exact sequences:

$$
\begin{gathered}
0 \rightarrow H \oplus Z_{3} \stackrel{\iota}{\rightarrow} \Lambda \stackrel{\eta}{\rightarrow} \bar{\Lambda} \rightarrow 0, \\
0 \rightarrow \Lambda \stackrel{\iota}{\rightarrow} \Gamma \oplus X_{3} \stackrel{\left[f-f_{3}\right]}{\rightarrow} \bar{\Lambda} \rightarrow 0,
\end{gathered}
$$

where $l$ denotes inclusion, $\eta$ the canonical map and the map $\left[f-f_{3}\right]$ is defined by $\left[f-f_{3}\right]\left(\gamma, x_{3}\right)=f(\gamma)-f_{3}\left(x_{3}\right)$. Now applying the functor $(-)^{*}=$ $\operatorname{Hom}_{R}(-, R)$ yields:

$$
(\mathrm{A})^{*} \quad 0 \rightarrow \Lambda^{*} \stackrel{\iota^{*}}{\rightarrow} H^{*} \oplus Z_{3}^{*} \stackrel{\partial}{\rightarrow} \operatorname{Ext}_{R}(\bar{\Lambda}, R)=: W \rightarrow 0,
$$




$$
0 \rightarrow \Gamma^{*} \oplus X_{3}^{*} \stackrel{\iota^{*}}{\rightarrow} \Lambda^{*} \stackrel{\partial}{\rightarrow} \operatorname{Ext}_{R}(\bar{\Lambda}, R)=: W \rightarrow 0,
$$

where $\partial$ is the boundary map from the long exact Hom sequence. This dual functor is a bijection between the category of right $\Lambda$-lattices and the category of left $\Lambda$-lattices. Consequently,

$$
W \cong \frac{\Lambda^{*}}{\Gamma^{*} \oplus X_{3}^{*}} \cong \frac{H^{*} \oplus Z_{3}^{*}}{\Lambda^{*}}
$$

and is an Artinian, uniserial, cyclic, left module of length 2 .

Since $H=\operatorname{pbk}\left(g_{1}: Y_{1} \rightarrow U \leftarrow Y_{2}: g_{2}\right)$, then $Z_{1} \oplus Z_{2}$ is the maximal submodule of $H$ where $Z_{i}$ is maximal in $Y_{i}$. Using a similar approach for $H$, we get the following exact sequences:

$$
\begin{aligned}
& 0 \rightarrow Z_{1} \oplus Z_{2} \stackrel{\iota}{\rightarrow} H \stackrel{\nu}{\rightarrow} U \rightarrow 0, \\
& 0 \rightarrow H \stackrel{\iota}{\rightarrow} Y_{1} \oplus Y_{2} \stackrel{\left[g_{1}-g_{2}\right]}{\rightarrow} U \rightarrow 0,
\end{aligned}
$$

where $\nu$ is the canonical map and $\left[g_{1}-g_{2}\right]\left(y_{1}, y_{2}\right)=g_{1}\left(y_{1}\right)-g_{2}\left(y_{2}\right)$. Applying the ${ }^{*}$-functor, we get

$$
\begin{aligned}
& 0 \rightarrow H^{*} \stackrel{\iota^{*}}{\rightarrow} Z_{i}^{*} \oplus Z_{2}^{*} \stackrel{\partial}{\rightarrow} \operatorname{Ext}_{R}(U, R) \rightarrow 0, \\
& 0 \rightarrow Y_{1}^{*} \oplus Y_{2}^{*} \stackrel{\iota^{*}}{\rightarrow} H^{*} \stackrel{\partial}{\rightarrow} \operatorname{Ext}_{R}(U, R) \rightarrow 0 .
\end{aligned}
$$

Thus,

$$
\operatorname{Ext}_{R}(U, R) \cong \frac{H^{*}}{Y_{1}^{*} \oplus Y_{2}^{*}} \cong \frac{Z_{1}^{*} \oplus Z_{2}^{*}}{H^{*}}
$$

is an Artinian, simple left $\Lambda$-module and so is isomorphic to $U$; identify $\operatorname{Ext}_{R}(U, R)$ with $U$.

Set $\mathfrak{X}_{i}=: Z_{i}^{*}$ which is a uniform left $\Lambda$-lattice.

Next we claim that $H^{*}=\operatorname{pbk}\left(s_{1}: \mathfrak{X}_{1} \rightarrow U \leftarrow \mathfrak{X}_{2}: s_{2}\right)$ for some epimorphisms $s_{i} \quad(i=1,2)$ such that $\operatorname{ker} s_{i}=Y_{i}^{*}$. Since $H^{*}$ is maximal in $\mathfrak{X}_{1} \oplus \mathfrak{X}_{2}$ and since $H^{*}$ is a full $\Lambda$-module of $K \mathfrak{X}_{1} \oplus K \mathfrak{X}_{2}$, then $H^{*}$ is a subdirect sum of $\mathfrak{X}_{1} \oplus \mathfrak{X}_{2}$. By Lemma 1.3 , write $H^{*}=\operatorname{pbk}\left(s_{1}: \mathfrak{X}_{1} \rightarrow \mathscr{V} \nleftarrow \mathfrak{X}_{2}: s_{2}\right)$ for some artinian left $\Lambda$-module $\mathscr{V}$ and epimorphisms $s_{1}$ and $s_{2}$. But $Y_{1}^{*} \oplus Y_{2}^{*} \subset H^{*}$ and so $Y_{i}^{*} \subset \operatorname{ker} s_{i}$. Now if $\operatorname{ker} s_{i}=\mathfrak{X}_{i}$, then $H^{*}$ (and so $H$ ) decomposes, a contradiction. Hence, $\operatorname{ker} s_{i} \neq \mathfrak{X}_{i}$. Yet $Y_{i}^{*}$ is maximal in $\mathfrak{X}_{i}\left(=Z_{i}^{*}\right)$ so we have $\operatorname{ker} s_{i}=Y_{i}^{*}$. Hence, $\mathscr{V} \cong \mathfrak{X}_{i} / Y_{i}^{*} \cong U$ and this proves the claim.

In a similar fashion, $\Lambda^{*}$ is also a subdirect sum of $H^{*}$ and $\mathfrak{X}_{3}$ so we can write $\Lambda^{*}=\operatorname{pbk}\left(s: H^{*} \rightarrow \mathscr{W} \leftarrow \mathfrak{X}_{3}: s_{3}\right)$. From $(\mathbf{B})^{*}, \Gamma^{*} \oplus X_{3}^{*} \subset \Lambda^{*}$; and so $\Gamma^{*} \subset \operatorname{ker} s \subset H^{*}$ (by (2) of Lemma 1.3). Similarly, we have $X_{3}^{*} \subset \operatorname{ker} s_{3} \subset \mathfrak{X}_{3}$. Now $2=l_{\Lambda}\left(\mathfrak{X}_{3} / X_{3}^{*}\right)$ (since $\left.2=l_{\Lambda}\left(X_{3} / Z_{3}\right)\right)$ and so $2 \geq l_{\Lambda}(\mathscr{W})$.

We claim that $l_{\Lambda}(\mathscr{W})=2$. If not, then $\mathscr{W} \cong U$ or 0 . If $\mathscr{W}=0$, then $\Lambda^{*}=H^{*} \oplus \mathfrak{X}_{3}$. This implies that $\Lambda \cong \Lambda^{* *} \cong H \oplus Z_{3}$, a contradiction since $\Lambda$ is local. If $\mathscr{W} \cong U$, then we have

$$
0 \rightarrow \Lambda^{*} \rightarrow H^{*} \oplus Z_{3}^{*} \rightarrow U \rightarrow 0 .
$$


Comparing this to sequence $(\mathrm{A})^{*}$, we see that $W \cong\left(H^{*} \oplus Z_{3}^{*}\right) / \Lambda^{*} \cong U$; this is a contradiction since $W$ has length 2 . Thus, $l_{\Lambda}(\mathscr{W})=2=l_{\Lambda}(W)$ (in fact, $\mathscr{W} \cong W$.

To complete the proof that $\Lambda^{*}$ is a left special quasi-triad, we must show that

$$
\operatorname{ker}\left(s: H^{*} \rightarrow \mathscr{W}\right)=\operatorname{pbk}\left(h_{1}: Y_{1}^{*} \rightarrow U \leftarrow Y_{2}^{*}: h_{2}\right)
$$

for some epimorphisms $h_{1}$ and $h_{2}$. We have seen that $\Gamma^{*} \subset \operatorname{ker} s$ (from $\left.(\mathrm{B})^{*}\right)$; yet $2=l_{\Lambda}(\mathscr{W})=l_{\Lambda}\left(H^{*} / \operatorname{ker} s\right)=l_{\Lambda}(W)=l_{\Lambda}\left(H^{*} / \Gamma^{*}\right)$, so we get that $\operatorname{ker} s=\Gamma^{*}$. Finally, using similar homological arguments as above, we observe that $\Gamma^{*}=\operatorname{pbk}\left(h_{1}: Y_{1}^{*} \rightarrow U \nleftarrow Y_{2}^{*}: h_{2}\right)$, as desired.

(3) $\Rightarrow$ (1) Using 1.3, it is straightforward to show that the triad $T=\Lambda+$ $\operatorname{rad} \widetilde{\Lambda}$ is minimal over $\Lambda$. Since $\Lambda$ is Gorenstein, then $T$ is the unique minimal overmodule of $\Lambda$.

While the radical of a triad is quite tractable $\left(\operatorname{rad} \Lambda=\operatorname{rad} \tilde{\Lambda}=Y_{1} \oplus Y_{2} \oplus Y_{3}\right)$, such is not the case for the special quasi-triad. The next lemma, however, shows some nice properties.

2.13 Lemma (Special quasi-triad facts). Let $\Lambda$ be a special quasi-triad and $T$ the associated triad using the notation of 2.12. Let $Z_{i}$ be the maximal submodule of $Y_{i}$. Then

(1) $\operatorname{ker}\left(\Gamma \rightarrow \bar{\Lambda}_{\Lambda}\right)=\left(y_{1}, y_{2}\right) \cdot T+Z_{1} \oplus Z_{2}$, where $y_{i}$ is some generator of $Y_{i}$. (In particular, this kernel is a T-module.)

(2) $\operatorname{rad} \Lambda=\left(y_{1}, 0, y_{3}\right) \cdot T+\left(0, y_{2}, y_{3}\right) \cdot T+Z_{1} \oplus Z_{2} \oplus Z_{3}$ is a (right) ideal of $T$.

(3) The results of (1) and (2) also hold for the left module structure of $\Lambda$. In particular, $\operatorname{rad} \Lambda$ is a 2-sided ideal of $T$.

Proof. (1) By Definition 2.9,

$$
H=\operatorname{ker}(f: \Gamma \rightarrow \bar{\Lambda})=\operatorname{pbk}\left(g_{1}: Y_{1} \rightarrow U \nleftarrow Y_{2}: g_{2}\right) .
$$

Let $y_{1}$ be some generator of $Y_{1}$. Since $U=\Lambda / \operatorname{rad} \Lambda$ is simple, there is a generator $y_{2}$ of $Y_{2}$ such that $\left(y_{1}, y_{2}\right) \in H$. We claim that $H=\left(y_{1}, y_{2}\right)$. $T+\left(Z_{1} \oplus Z_{2}\right)$, where $Z_{i}$ is the maximal submodule of $Y_{i}$. If $x \in H$, then $x=\left(y_{1} \lambda, y_{2} \tau\right)$ such that $g_{1}\left(y_{1} \lambda\right)=g_{2}\left(y_{2} \tau\right)$. But $g_{1}\left(y_{1}\right)=g_{2}\left(y_{2}\right)$ and so $y_{2} \lambda-y_{2} \tau \in \operatorname{ker} g_{2}=Z_{2}$. Hence

$$
x=\left(y_{1}, y_{2}\right) \lambda-\left(0, y_{2}\right) \lambda+\left(0, y_{2}\right) \tau \in\left(y_{1}, y_{2}\right) T+0 \oplus Z_{2}
$$

which proves the claim.

(2) Let $M=\operatorname{rad} \Lambda$. From the right regular module structure of $\Lambda$, the projection of $M$ into $\Gamma$ is the maximal submodule of $\Gamma$, namely $Y_{1} \oplus Y_{2}$, while the projection of $M$ into $X_{3}$ is $Y_{3}$. The pullback structure for $M$ is

$$
M=\operatorname{pbk}\left(m: Y_{1} \oplus Y_{2} \rightarrow \bar{M} \leftarrow Y_{3}: m_{3}\right),
$$

where $\bar{M}=M / \operatorname{ker}(\eta: \Lambda \rightarrow \bar{\Lambda}) \cong \Lambda / \operatorname{rad} \Lambda$ and $\operatorname{ker} m=\operatorname{ker}(f: \Gamma \rightarrow \bar{\Lambda})=H=$ $\left(y_{1}, y_{2}\right) \cdot T+Z_{1} \oplus Z_{2}$ which is maximal in $Y_{1} \oplus Y_{2}$. If $y_{3}$ is a generator of $Y_{3}$, 
then there exists generators $y_{1}$ and $y_{2}$ of $Y_{1}$ and $Y_{2}$ respectively such that $\left(y_{1}, 0, y_{3}\right) \in M$ and $\left(0, y_{2}, y_{3}\right) \in M$. If $x \in M$, then $x=\left(y_{1} \lambda, y_{2} \tau, y_{3} \sigma\right)$ where $m\left(y_{1} \lambda, y_{2} \tau\right)=m_{3}\left(y_{3} \sigma\right)$ and $\lambda, \tau, \sigma \in \Lambda \subset T$. But

$$
m\left(y_{1} \lambda, y_{2} \tau\right)=m\left(y_{1} \lambda, 0\right)+m\left(0, y_{2} \tau\right)=m_{3}\left(y_{3}\right) \lambda+m_{3}\left(y_{3}\right) \tau
$$

so that $y_{3}(\sigma-\lambda-\tau) \in \operatorname{ker} m_{3}=Z_{3}$. Hence,

$$
\begin{aligned}
x & =\left(y_{1}, 0, y_{3}\right) \lambda+\left(0, y_{2}, y_{3}\right) \tau+\left(0,0, y_{3}(\sigma-\lambda-\tau)\right) \\
& \in\left(y_{1}, 0, y_{3}\right) \cdot T+\left(0, y_{2}, y_{3}\right) \cdot T+Z_{1} \oplus Z_{2} \oplus Z_{3},
\end{aligned}
$$

as desired.

(3) This follows from symmetric arguments.

The following result of [DK'73], stated without proof, plays a crucial role in proving Theorem 2.1.

2.14 Theorem [DK'73, 8.1]. Let $T$ be a local, non-Gorenstein order of FRT with $l_{A}(A)=3$. Then $T$ is sigma-I if and only if $\operatorname{rad} T$ is an ideal of $\widetilde{T}$.

Before proving Theorem 2.1, we will characterize all local sigma- $I$ orders such that $l_{A}(A)=3$.

2.15 Theorem. Let $R, K, \Lambda$ and $A$ be as in 2.1 such that $l_{A}(A)=3$. Then $\Lambda$ is sigma-I if and only if $\Lambda$ is either

(a) a triad, or

(b) a special quasi-triad.

Proof. ( $\Rightarrow$ ) if $\Lambda$ is a sigma-I order then $\Lambda$ has FRT by 2.3(1). Subsequently, either $\Lambda$ is non-Gorenstein with $\operatorname{rad} \Lambda$ an ideal of $\tilde{\Lambda}$ or else $\Lambda$ is Gorenstein with minimal overorder $T$ which is local, non-Gorenstein and sigma- $I$ (2.8).

Suppose $\Lambda$ is non-Gorenstein and so $\operatorname{rad} \Lambda$ is a 2-sided $\widetilde{\Lambda}$-ideal. But from 2.3, $\widetilde{\Lambda}=X_{1} \oplus X_{2} \oplus X_{3}$ where $\left\{X_{i}\right\}$ is a complete set of isomorphism representatives of uniform right $\Lambda$-lattices as well as the indecomposable projective $\tilde{\Lambda}$-modules. Set $\bar{X}_{i}=X_{i} /\left(X_{i} \cdot \operatorname{rad} \Lambda\right)$ and so $\Theta=: \tilde{\Lambda} / \operatorname{rad} \Lambda=\bar{X}_{1} \oplus \bar{X}_{2} \oplus \bar{X}_{3}$. However, by 2.3 , each $X_{i}$ is cyclic and hence the set $\left\{\bar{X}_{i}\right\}$ consists of all the simple $\Lambda$ and $\tilde{\Lambda}$ modules. Thus, $\Theta$ is semisimple Artinian so that $\operatorname{rad} \tilde{\Lambda} \subset \operatorname{rad} \Lambda$. The opposite inclusion is known from 2.5. This shows that $\Lambda$ is a local order such that $\operatorname{rad} \Lambda=\operatorname{rad} \tilde{\Lambda}$ and so $\Lambda$ is the triad.

Now suppose that $\Lambda$ is Gorenstein with minimal overorder $T$ which is nonGorenstein, local and sigma- $I$. From the above paragraph, $T$ is the triad and so $\Lambda$ is a special quasi-triad.

$(\Leftrightarrow)$ First suppose $\Lambda$ is a triad. Then from $2.8, \Lambda$ is non-Gorenstein with $\operatorname{rad} \Lambda=\operatorname{rad} \widetilde{\Lambda}$, an ideal of $\widetilde{\Lambda}$. By $2.14, \Lambda$ is sigma- $I$.

Now suppose $\Lambda$ is a special quasi-triad. Then $T=\Lambda+\operatorname{rad} \tilde{\Lambda}$ is the unique minimal overmodule of $\Lambda$, so, by Theorem 2.8, $T$ is non-Gorenstein and sigma- $I$. From (4) of Theorem 2.8, every indecomposable $\Lambda$-lattice is either 
$\Lambda$ or some indecomposable $T$-lattice. Since $T$ is sigma- $I$, then so is $\Lambda$. This completes the proof.

Historically, Bass [B] showed that, in the commutative case, if $\Lambda$ is sigma- $I$ then $P_{i}+P_{j}=\operatorname{rad} \Lambda$. This is also the case in the noncommutative situation as seen by the next result.

2.16 Corollary. Let $R, K, \Lambda$ and $A$ be as in 2.1 such that $l_{A}(A)=3$. If $\Lambda$ is sigma-I, then $P_{i}+P_{j}=\operatorname{rad} \Lambda=Q_{i}+Q_{j}$ for all $i, j \in\{1,2,3\}, i \neq j$.

Proof. If $\Lambda$ is the triad, observe that $P_{k}=Y_{i} \oplus Y_{j}$ since $\operatorname{rad} \Lambda=Y_{1} \oplus Y_{2} \oplus Y_{3}$. Symmetrically, $Q_{i}+Q_{j}=\operatorname{rad} \Lambda$ for any $i \neq j$.

If $\Lambda$ is the special quasi-triad, then by Lemma 2.13, $\operatorname{rad} \Lambda=\left(y_{1}, 0, y_{3}\right) \cdot T+$ $\left(0, y_{2}, y_{3}\right) \cdot T+Z_{1} \oplus Z_{2} \oplus Z_{3}$. From inspection, $P_{1}=\left(0, y_{2}, y_{3}\right) \cdot T+0 \oplus Z_{2} \oplus Z_{3}$ and $P_{2}=\left(y_{1}, 0, y_{3}\right) \cdot T+Z_{1} \oplus 0 \oplus Z_{3}$. Now $P_{3} \subset \operatorname{rad} \Lambda$ so $P_{3}=\left(y_{1},-y_{2}, 0\right)$. $T+Z_{1} \oplus Z_{2} \oplus 0$. In particular, $P_{i}+P_{j}=\operatorname{rad} \Lambda$ for any $i \neq j$. Symmetrically, $Q_{i}+Q_{j}=\operatorname{rad} \Lambda$ for any $i \neq j$.

Finally, the proof of Theorem 2.1 is at hand.

Proof of 2.1. $(\Rightarrow)$ Using Proposition 2.3, we see that if $\Lambda$ is sigma- $I$, then $\Lambda$ has FRT so $l_{A}(A) \leq 3$. If $l_{A}(A) \leq 2$, then $\Lambda$ is a local Bass order by $2.3(2)$. If $l_{A}(A)=3$, then $\Lambda$ is either a triad or a special quasi-triad by 2.15 .

$(\Leftarrow)$ Every local Bass order is sigma- $I$ from [DKR, 12.1] and Theorem 2.15 shows that every triad and special quasi-triad are sigma- $I$.

An appropriate remark is that the sigma- $I$ property is left-right symmetric. Indeed, this can be directly verified by dualizing with respect to $R$; that is, $M$ is a right indecomposable $\Lambda$-lattice if and only if $M^{*}=\operatorname{Hom}_{R}(M, R)$ is an indecomposable left $\Lambda$-lattice.

\section{EXAMPLES}

In this section, we provide some examples of sigma- $I$ (but not Bass) rings to elucidate the notions of triad and special quasi-triad. In certain cases, we can give a definitive form for the triad.

If $\Lambda$ is a local sigma- $I$ order which is not Bass then $l_{A}(A)=3$ and so there are three possibilities for the separable algebra $A: M_{3}(D), M_{2}(D) \oplus D_{1}$ or $D_{1} \oplus D_{2} \oplus D_{3}$ where $D, D_{1}, D_{2}, D_{3}$ are division rings. Let $\mathscr{D}$ (respectively $\mathscr{D}_{i}$ ) be the unique maximal $R$-order in $D\left(D_{i}\right)$, let $\mathscr{P}=\pi \mathscr{D}=\mathscr{D} \pi$ (respectively, $\left.\mathscr{P}_{i}=\pi \mathscr{D}_{i}=\mathscr{D}_{i} \pi\right)$ be the unique maximal ideal of $\mathscr{D}\left(\mathscr{D}_{i}\right)$ and let $k$ (respectively, $k_{i}$ ) be the residue division ring. Assume throughout this section that $\Lambda$ is a local $R$-order in $A$ with FRT.

The case $A=M_{3}(D)$. Since $R$ is a complete local Dedekind domain, any hereditary order inside $A$ is, up to isomorphism, one of the following:

$$
\left[\begin{array}{lll}
\mathscr{D} & \mathscr{D} & \mathscr{D} \\
\mathscr{P} & \mathscr{D} & \mathscr{D} \\
\mathscr{P} & \mathscr{P} & \mathscr{D}
\end{array}\right], \quad\left[\begin{array}{lll}
\mathscr{D} & \mathscr{D} & \mathscr{D} \\
\mathscr{D} & \mathscr{D} & \mathscr{D} \\
\mathscr{P} & \mathscr{P} & \mathscr{D}
\end{array}\right] \quad \text { and } \quad M_{3}(\mathscr{D}) .
$$


The second possibility is ruled out for $\tilde{\Lambda}$ by [DK'73, 3.1]. Let $I$ denote the $3 \times 3$ matrix identity:

3.1 Proposition. (1) If

$$
\widetilde{\Lambda}=\left[\begin{array}{lll}
\mathscr{D} & \mathscr{D} & \mathscr{D} \\
\mathscr{P} & \mathscr{D} & \mathscr{D} \\
\mathscr{P} & \mathscr{P} & \mathscr{D}
\end{array}\right]
$$

then the triad $\Lambda$ has the form

$$
\Lambda=\left[\begin{array}{lll}
\mathscr{P} & \mathscr{D} & \mathscr{D} \\
\mathscr{P} & \mathscr{P} & \mathscr{D} \\
\mathscr{P} & \mathscr{P} & \mathscr{P}
\end{array}\right]+I \cdot \mathscr{D} .
$$

(2) If $\tilde{\Lambda}=M_{3}(\mathscr{D})$, then the triad $\Lambda$ has the form $\Lambda=\left\langle I, \mathbf{x}_{2}, \mathbf{x}_{3}\right\rangle+M_{3}(\mathscr{P})$, where $\left\langle I, \mathbf{x}_{2}, \mathbf{x}_{3}\right\rangle$ is the subring of $\tilde{\Lambda}$ generated by $I, \mathbf{x}_{2}$ and $\mathbf{x}_{3}$ such that, modulo $M_{3}(\mathscr{P}),\left\langle I, \mathbf{x}_{2}, \mathbf{x}_{3}\right\rangle$ generates a division subring in $M_{3}(k)$ of $k$-dimension 3 .

Conversely, $\left\langle I, \mathbf{x}_{2}, \mathbf{x}_{3}\right\rangle+M_{3}(\mathscr{P})$ is a triad provided $\left\langle I, \mathbf{x}_{2}, \mathbf{x}_{3}\right\rangle$ modulo $M_{3}(\mathscr{P})$ forms a division ring of $k$-dimension 3 .

Proof. (1) It is easy to verify that $\Lambda$ is a ring with

$$
\operatorname{rad} \Lambda=\left[\begin{array}{ccc}
\mathscr{P} & \mathscr{D} & \mathscr{D} \\
\mathscr{P} & \mathscr{P} & \mathscr{D} \\
\mathscr{P} & \mathscr{P} & \mathscr{P}
\end{array}\right]=\operatorname{rad} \tilde{\Lambda}
$$

and so $\Lambda$ is a triad. Suppose $T$ is another triad contained in $\widetilde{\Lambda}$. Then $\operatorname{rad} T=$ $\operatorname{rad} \tilde{\Lambda}=\operatorname{rad} \Lambda$. But $T$ is a $\mathscr{D}$-module and $I \in T$ so $\Lambda \subset T \subset \widetilde{\Lambda}$. Notice that $X_{1}=(\mathscr{D} \mathscr{D} \mathscr{D}), X_{2}=(\mathscr{P} \mathscr{D} \mathscr{D})$ and $X_{3}=(\mathscr{P} \mathscr{P} \mathscr{D})$ with maximal submodules (respectively) $Y_{1}=(\mathscr{P} \mathscr{D} \mathscr{D}), \quad Y_{2}=(\mathscr{P} \mathscr{P} \mathscr{D}), \quad Y_{3}=(\mathscr{P} \mathscr{P} \mathscr{P})$. Now we have $X_{i} / Y_{i} \cong \Lambda / \operatorname{rad} \Lambda \subset T / \operatorname{rad} \Lambda=T / \operatorname{rad} T \cong X_{i} / Y_{i}$ and these are simple $\Lambda$-modules. Thus, if $\Lambda / \operatorname{rad} \Lambda$ and $T / \operatorname{rad} \Lambda$ are simple $\Lambda$-modules, then $T=\Lambda$.

(2) Now $M_{3}(\mathscr{P}) \subset \operatorname{rad} \Lambda$ (by exercise 3, p. 365 of $[\mathrm{R}]$ ). Further, $M_{3}(\mathscr{P})$ is a 2-sided $\tilde{\Lambda}$-ideal such that $\Lambda / M_{3}(\mathscr{P})$ is a division ring. As a result, the ring $\Lambda$ is local. Hence, $\operatorname{rad} \Lambda=M_{3}(\mathscr{P})=\operatorname{rad} \tilde{\Lambda}$ and so $\Lambda$ is a triad. In this case, the uniform lattices are all isomorphic so set $X_{i}=(\mathscr{D} \mathscr{D} \mathscr{D})$ with maximal submodule $Y_{i}=(\mathscr{P} \mathscr{P} \mathscr{P})$.

If $T$ is any other triad in $\tilde{\Lambda}$, then

$$
\bar{T}=T / \operatorname{rad} T \cong X_{i} / Y_{i} \cong(\mathscr{D} \mathscr{D} \mathscr{D}) /(\mathscr{P} \mathscr{P} \mathscr{P}) \cong(k k k) \text {. }
$$

Hence $\bar{T}$ is a division ring with $k$-dimension 3. Let $I, \overline{\mathbf{y}_{2}}$ and $\overline{\mathbf{y}_{3}}$ generate $\bar{T}$ as a right $k$-module. Lift these to $I, \mathbf{y}_{2}$ and $\mathbf{y}_{3}$ in $\tilde{\Lambda}$ and put $\Lambda=\left\langle I, \mathbf{y}_{2}, \mathbf{y}_{3}\right\rangle+$ $M_{3}(\mathscr{P})$ so that $\Lambda \subset T$. But $\Lambda$ is a triad such that $\Lambda / \operatorname{rad} \Lambda=T / \operatorname{rad} \Lambda=$ $\Lambda / \operatorname{rad} \Lambda$. This forces $T=\Lambda$.

3.2 Examples. (1) This first example illustrates the triad of 3.1(2). Let $R$ be a local (commutative) Dedekind domain with maximal ideal $P$ and residue 
field $k=R / P$ and suppose $F$ is a field extension of $k$ of degree 3. Then $F$ embeds into $A=M_{3}(k)$ such that $|A: F|=|F: k|=3$. Let $F$ be generated, as a $k$-algebra, by $I, \overline{\mathbf{x}}_{2}$ and $\overline{\mathbf{x}}_{3}$ in $A$ and lift these to $M_{3}(R)$. Now define $T$ as in 3.1(2).

(2) For an example of a special quasi-triad inside $A=M_{3}(D)$, let

$$
T=\left[\begin{array}{ccc}
\mathscr{P} & \mathscr{D} & \mathscr{D} \\
\mathscr{P} & \mathscr{P} & \mathscr{D} \\
\mathscr{P} & \mathscr{P} & \mathscr{P}
\end{array}\right]+I \cdot \mathscr{D}
$$

be the triad as in 3.1(1). Observe that

$$
\begin{gathered}
Y_{1}=\left[\begin{array}{ccc}
\mathscr{P} & \mathscr{D} & \mathscr{D} \\
0 & 0 & 0 \\
0 & 0 & 0
\end{array}\right]=\left[\begin{array}{lll}
0 & 1 & 0 \\
0 & 0 & 0 \\
0 & 0 & 0
\end{array}\right] \cdot T=\left(y_{1}, 0,0\right) \cdot T, \\
Y_{2}=\left[\begin{array}{ccc}
0 & 0 & 0 \\
\mathscr{P} & \mathscr{P} & \mathscr{D} \\
0 & 0 & 0
\end{array}\right]=\left[\begin{array}{lll}
0 & 0 & 0 \\
0 & 0 & 1 \\
0 & 0 & 0
\end{array}\right] \cdot T=\left(0, y_{2}, 0\right) \cdot T
\end{gathered}
$$

and

$$
Y_{3}=\left[\begin{array}{ccc}
0 & 0 & 0 \\
0 & 0 & 0 \\
\mathscr{P} & \mathscr{P} & \mathscr{P}
\end{array}\right]=\left[\begin{array}{lll}
0 & 0 & 0 \\
0 & 0 & 0 \\
\pi & 0 & 0
\end{array}\right] \cdot T=\left(0,0, y_{3}\right) \cdot T,
$$

where $y_{1}=(010), y_{2}=\left(\begin{array}{ll}0 & 01\end{array}\right)$ and $y_{3}=(\pi 00)$. The maximal submodule of $Y_{i}$ is $Z_{i}$ so following $2.13(2)$, set

$$
\begin{aligned}
M & =\left(y_{1}, 0, y_{3}\right) \cdot T+\left(0, y_{2}, y_{3}\right) \cdot T+Z_{1} \oplus Z_{2} \oplus Z_{3} \\
& =\left[\begin{array}{lll}
0 & 1 & 0 \\
0 & 0 & 0 \\
\pi & 0 & 0
\end{array}\right] \cdot T+\left[\begin{array}{lll}
0 & 1 & 0 \\
0 & 0 & 1 \\
0 & 0 & 0
\end{array}\right] \cdot T+\left[\begin{array}{ccc}
\mathscr{P} & \mathscr{P} & \mathscr{D} \\
\mathscr{P} & \mathscr{P} & \mathscr{P} \\
\mathscr{P}^{2} & \mathscr{P} & \mathscr{P}
\end{array}\right] .
\end{aligned}
$$

It is straightforward to check that $\Lambda=M+I \cdot \mathscr{D}$ is a local order such that $\operatorname{rad} \Lambda=M$. In addition, $T$ is uniquely minimal over $\Lambda$. By $2.12, \Lambda$ is a special quasi-triad and is sigma- $I$.

The case $A=M_{2}(D) \oplus D_{1}$. Assume $k \cong k_{1}$ as rings. As in the case above, the hereditary order $\tilde{\Lambda}$ is isomorphic to either

$$
\left[\begin{array}{ll}
\mathscr{D} & \mathscr{D} \\
\mathscr{D} & \mathscr{D}
\end{array}\right] \oplus \mathscr{D}_{1} \quad \text { or } \quad M_{2}(\mathscr{D}) \oplus \mathscr{D}_{1} \text {. }
$$

3.3 Proposition. (1) If

$$
\tilde{\Lambda}=\left[\begin{array}{ll}
\mathscr{D} & \mathscr{D} \\
\mathscr{P} & \mathscr{D}
\end{array}\right] \oplus \mathscr{D}_{1},
$$

then the triad $\Lambda \subset \tilde{\Lambda}$ has the form

$$
\Lambda=\operatorname{pbk}\left(f: \Gamma \rightarrow k \cong k_{1} \leftarrow \mathscr{D}_{1}: f_{3}\right)
$$

(as a ring), where $\Gamma=\left[\begin{array}{cc}\mathscr{P} & \mathscr{D} \\ \mathscr{P} & \mathscr{P}\end{array}\right]+I \cdot \mathscr{D}$. 
(2) If $\tilde{\Lambda}=M_{2}(\mathscr{D}) \oplus \mathscr{D}_{1}$, then the triad $\Lambda$ has the form

$$
\Lambda=\operatorname{pbk}\left(f: \Gamma \rightarrow \bar{U} \leftarrow \mathscr{D}_{1}: f_{3}\right)
$$

(as a ring), where $\Gamma=\operatorname{pbk}\left(f_{1}:(\mathscr{D} \mathscr{D}) \rightarrow U \leftarrow(\mathscr{D} \mathscr{D}): f_{2}\right)$ and $U=\Lambda / \operatorname{rad} \Lambda \cong$ $\Gamma / \operatorname{rad} \Gamma \cong \mathscr{D}_{1} / \mathscr{P}_{1}$ is a 2-dimensional skewfield extension of $k=\mathscr{D} / \mathscr{P}$.

Also, $\operatorname{ker} f=M_{2}(\mathscr{P}), \operatorname{ker} f_{3}=\mathscr{P}_{3}$ and $\operatorname{ker} f_{1} \cong \operatorname{ker} f_{2} \cong(\mathscr{P} \mathscr{P})$.

Proof. (1) Let $\Lambda$ be as above. Since the maps in the pullback are ring homo-

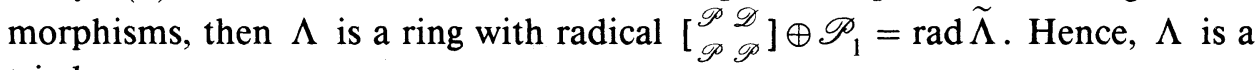
triad.

If $T$ is also a triad then $\operatorname{rad} T=\operatorname{rad} \Lambda=\operatorname{rad} \tilde{\Lambda}$. Write

$$
T_{T}=\operatorname{pbk}\left(t: \Theta \rightarrow U \leftarrow \mathscr{D}_{1}: t_{3}\right)
$$

using the notation of 2.11 and the fact that $X_{3}=\mathscr{D}_{1}$. Now $U=T / \operatorname{rad} T=$ $T / \operatorname{rad} \tilde{\Lambda}$ is a division ring containing $k$ and sits inside $\tilde{\Lambda} / \operatorname{rad} \tilde{\Lambda} \cong k \oplus k \oplus k$. Furthermore, $\widetilde{\Lambda} / \operatorname{rad} \tilde{\Lambda}$ is a direct sum of three simple $T$-modules. This implies $U \cong k_{1} \cong k$. It is straightforward to check that, since $T$ is a triad, then $\Gamma=\Theta$ as rings and so $T \cong \Lambda$.

(2) If $\Lambda$ has the form of (2), then it is easy to see that

$$
\begin{aligned}
\operatorname{rad} \Lambda & =\operatorname{ker} f_{1} \oplus \operatorname{ker} f_{2} \oplus \operatorname{ker} f_{3}=(\mathscr{P} \mathscr{P}) \oplus(\mathscr{P} \mathscr{P}) \oplus \mathscr{P}_{1} \\
& =M_{2}(\mathscr{P}) \oplus \mathscr{P}_{1}=\operatorname{rad} \tilde{\Lambda} .
\end{aligned}
$$

Conversely, suppose $T$ is a triad. Then using 2.11 , write

$$
T_{T}=\operatorname{pbk}\left(t: \Theta \rightarrow U \leftarrow \mathscr{D}_{1}: t_{3}\right)
$$

where $U=T / \operatorname{rad} T$. In this case, $X_{1} \cong X_{2} \cong(\mathscr{D} \mathscr{D}), X_{3}=\mathscr{D}_{1}, Y_{1} \cong$ $Y_{2} \cong(\mathscr{P} \mathscr{P})$ and $Y_{3}=\mathscr{P}_{1}$ are the right uniforms while $\mathfrak{X}_{1} \cong \mathfrak{X}_{2} \cong\left[\mathscr{D}_{\mathscr{D}}\right]$, $\mathfrak{X}_{3}=\mathscr{D}_{1}, \mathscr{Y}_{1} \cong \mathscr{Y}_{2} \cong\left[\begin{array}{l}\mathscr{P} \\ \mathscr{P}\end{array}\right]$ and $\mathscr{Y}_{3}=\mathscr{P}_{1}$ are the left uniforms. In particular, $U=T / \operatorname{rad} T \cong X_{1} / Y_{1} \cong(k k)$ is a 2-dimensional $k$-space. Now by Remark 2.7 ,

$$
\Theta=\operatorname{pbk}\left(t_{1}:(\mathscr{D} \mathscr{D}) \rightarrow U \leftarrow(\mathscr{D} \mathscr{D}): t_{2}\right)
$$

and is the projection of $\Lambda$ into $M_{2}(\mathscr{D})$; hence $\Theta$ is a local order. Furthermore, $\operatorname{rad} \Theta=M_{2}(\mathscr{P})$ so $\Theta / \operatorname{rad} \Theta \cong U$ as desired.

3.4 Examples. (1) Let $R, P$ and $k$ be as in Example 3.2(1). Then the order

$$
T=\left[\begin{array}{ll}
P & R \\
P & P
\end{array}\right] \oplus P+(I \oplus 1) \cdot R
$$

is local with

$$
\operatorname{rad} T=\left[\begin{array}{ll}
P & R \\
P & P
\end{array}\right] \oplus P=\operatorname{rad} \tilde{\Lambda}
$$

and so $T$ is a triad. 
(2) For an example of a special quasi-triad in an algebra of the form $M_{2}\left(K_{1}\right) \oplus$ $K_{2}$, let $R, P, k$ be as before with $P=R p=p R$. Define the ring $\Gamma=$ $\left[\begin{array}{ll}P & R \\ P & P\end{array}\right]+I \cdot R$. Notice that $\Gamma=\operatorname{pbk}\left(f_{1}: X_{1} \rightarrow k \nleftarrow X_{2}: f_{2}\right)$, where $X_{1} \cong(R R)$ and $X_{2} \cong(P R)$ are uniform right $\Gamma$-lattices. The maps $f_{1}$ and $f_{2}$ have kernels

$$
Y_{1}=\left[\begin{array}{ll}
0 & 1 \\
0 & 0
\end{array}\right] \cdot \Gamma=\left[\begin{array}{cc}
P & R \\
0 & 0
\end{array}\right] \cdot \Gamma \cong(P R)
$$

and

$$
Y_{2}=\left[\begin{array}{ll}
0 & 0 \\
p & 0
\end{array}\right] \cdot \Gamma=\left[\begin{array}{ll}
0 & 0 \\
P & P
\end{array}\right] \cdot \Gamma \cong(P P)
$$

respectively. Now set

$$
V=\Gamma /\left[\begin{array}{ll}
0 & 1 \\
p & 0
\end{array}\right] \cdot \Gamma
$$

and check that $V \cong\left\{\left[\begin{array}{ll}x & y \\ 0 & x\end{array}\right]\right\}$, where $x$ and $y$ belong to $k$; i.e., $V$ is a commutative, Artinian valuation ring of length 2 . Such a ring, by a theorem of Hungerford $[\mathrm{H}]$, is a homomorphic image of some principal ideal domain. A suitable localization of this PID yields the necessary discrete valuation ring $\mathscr{D}_{1}$ mapping onto $V$. Thus,

$$
\Lambda=\operatorname{pbk}\left(f: \Gamma \rightarrow V \nleftarrow \mathscr{D}_{1}: f_{3}\right)
$$

is a (noncommutative) special quasi-triad. Of course,

$$
T=\Lambda+\left\{\left[\begin{array}{ll}
P & R \\
P & P
\end{array}\right] \oplus \mathscr{P}_{1}\right\}=\Lambda+\operatorname{rad} \tilde{\Lambda}
$$

is a triad by 2.8 .

(3) For an example of a triad when $\tilde{\Lambda}=M_{2}(\mathscr{D}) \oplus \mathscr{D}_{1}$, set $\Omega=M_{2}\left(\mathbb{Z}_{3}\right)$, where $\mathbb{Z}_{3}$ is the localization of $\mathbb{Z}$ at 3 . Let $P=3 \cdot \mathbb{Z}_{3}$ be the unique maximal ideal of $\mathbb{Z}_{3}$. Define

$$
\Gamma=\left[\begin{array}{ll}
1 & 0 \\
0 & 1
\end{array}\right] \cdot \mathbb{Z}_{3}+\left[\begin{array}{ll}
0 & 1 \\
1 & 1
\end{array}\right] \cdot \mathbb{Z}_{3}+M_{2}(P)
$$

which is a local Bass order such that $\tilde{\Gamma}=\Omega$ and $\operatorname{rad} \Gamma=M_{2}(P)$. Note that $\Gamma / \operatorname{rad} \Gamma$ is a 2-dimensional field extension of $k=\mathbb{Z}_{3} / P \cong \mathbb{Z}$ modulo 3 (since $\sqrt{5} \notin \mathbb{Z} \bmod 3)$. Choose a discrete valuation ring $\mathscr{D}_{1}$ with maximal ideal $\mathscr{P}_{1}$ such that $\Gamma / \operatorname{rad} \Gamma \cong \mathscr{D}_{1} / \mathscr{P}_{1}$ (see $\left.[\mathrm{H}]\right)$. Define

$$
T=\operatorname{pbk}\left(t: \Gamma \rightarrow \Gamma / \operatorname{rad} \Gamma \nleftarrow \mathscr{D}_{1}: t_{3}\right)
$$

which is a local order in $\Omega \oplus \mathscr{D}_{1}=\widetilde{T}$. Note that $\operatorname{rad} T=M_{2}(P) \oplus \mathscr{P}_{1}=$ $\operatorname{rad}\left(\Omega \oplus \mathscr{D}_{1}\right)=\operatorname{rad} \widetilde{T}$. By 2.6, $T$ is a triad and so is sigma- $I$.

The case $A=D_{1} \oplus D_{2} \oplus D_{3}$. In this case, there is a unique maximal (and hereditary) $R$-order, $\Omega=\mathscr{D}_{1} \oplus \mathscr{D}_{2} \oplus \mathscr{D}_{3}$. Let $e_{1}, e_{2}$ and $e_{3}$ be the central primitive orthogonal idempotents in $\Omega$. 
3.5 Theorem. The following statements are equivalent for a local order $\Lambda$ in $A$ :

(1) $\Lambda$ is sigma-I.

(2) $\Lambda$ is either

(a) the triad of $\mathscr{D}_{1} \oplus \mathscr{D}_{2} \oplus \mathscr{D}_{3}$; that is, $\Lambda=\left\{\left(d_{1}, d_{2}, d_{3}\right)\right.$ : $f_{1} d_{1}=f_{2} d_{2}=$ $\left.f_{3} d_{3}\right\}$, where $f_{i}: \mathscr{D}_{i} \rightarrow k \cong \mathscr{D}_{i} / \mathscr{P}_{i}$, or

(b) the special quasi-triad; that is, $\Lambda=\operatorname{pbk}\left(f: \Gamma \rightarrow V \nleftarrow \mathscr{D}_{3}: f_{3}\right)$, where $\Gamma=\operatorname{pbk}\left(f_{1}: \mathscr{D}_{1} \rightarrow k \nleftarrow \mathscr{D}_{2}: f_{2}\right), V \cong \Gamma /\left(y_{1}+y_{2}\right) \cdot \Gamma$ and $y_{i}$ is some generator of $Y_{i}$.

(3) $P_{i}+P_{j}=\operatorname{rad} \Lambda$ for all $i \neq j$.

Proof. By Theorem 2.15 and Corollary 2.16, it suffices to show that (3) implies (2). Yet the $P_{i}$ are 2-sided ideals of $\Lambda$ so the same argument used in [HL, 3.6] (slightly modified for the noncommutativity of the $\mathscr{D}_{i}$ ) will work here.

Remarks. (1) The existence of a special quasi-triad depends on the ramification index of the maximal ideal $P$ of $R$ within the maximal orders $\mathscr{D}_{i}$. See [NR] for details.

(2) For examples of triads and special quasi-triads in the case $A=D_{1} \oplus D_{2} \oplus$ $D_{3}$, see [HL, $\left.\S 4\right]$.

\section{FinAL REMARKS}

Here are some remarks and questions for further investigation of sigma- $I$ orders.

(1) What happens to the sigma-I property when passing from local orders over complete local Dedekind domains to arbitrary orders over complete local Dedekind domains? A reduction theorem such as that found in [DK'72] might be possible.

(2) What is true about arbitrary orders over Dedekind domains having the sigma-I property? In this case, an analogous tool as the graph of the spectrum of the ring $R$ may help classify the sigma- $I$ orders.

(3) Klingler [K] has discovered an apparent flaw in a paper by Berman; in doing so, he was able to classify which integral group rings (for groups with square-free order) have the sigma-I property as well as describe the genera of lattices over such rings.

Acknowledgment. I would like to thank the referee for making many helpful comments for the revision of this paper.

\section{REFERENCES}

[B] H. Bass, On the ubiquity of Gorenstein rings, Math. Z. 82 (1963), 8-28.

[CR] C. W. Curtis and I. Reiner, Methods of representation theory, with applications to finite groups and orders, vol. 1, Wiley, New York, 1981.

[DK'72] J. A. Drozd and V. V. Kiricenko, On quasi-Bass orders, Izv. Akad. Nauk SSSR 36 (1972), no. 2; English transl., Math. USSR-Izv. 6 (1972), no. 2, 323-365.

[DK'73] _ _ Primary orders with a finite number of indecomposable representations, Izv. Akad. Nauk SSSR 37 (1973), no. 4; English transl., Math. USSR-Izv. 7 (1973), no. 4, 711-732. 
[DKR] J. A. Drozd, V. V. Kiricenko and A. V. Roiter, On hereditary and Bass orders, Izv. Akad. Nauk SSSR 31 (1967), no. 6; English transl., Math. USSR-Izv. 1 (1967), no. 6, 1357-1375.

[G] C. Greither, On the 2-generator problem for the ideals of a one-dimensional ring, J. Pure Appl. Algebra 24 (1982), 265-276.

[HL] J. Haefner and L. Levy, Commutative orders whose lattices are direct sums of ideals, J. Pure Appl. Algebra 50 (1988), 1-20.

[K] L. Klingler, Integral representations of groups of square free order, J. Algebra (to appear).

[NR] L. A. Nazarova and A. V. Roiter, Refinement of a theorem of Bass, Dokl. Akad. Nauk SSSR (1967), 262-268; English transl., Soviet Math. 8 (1967), 1089-1092.

[R] I. Reiner, Maximal orders, Academic Press, 1975.

[R'66] A. V. Roiter, An analog of the theorem of Bass for modules of representations of noncommmutative orders, Dokl. Akad. Nauk SSSR 168 (1966), 1261-1264; English transl., Soviet Math. Dokl. 7 (1966), 830-833.

[R'68] _ Unbounded dimensionality of indecomposable representations of an algebra with an infinite number of indecomposable representations, Izv. Akad. Nauk SSSR 32 (1968), no. 6; English transl., Math. USSR-Izv. 2 (1968), no. 6, 1223-1230.

Department of Mathematics, University of Tennessee, Knoxville, Tennessee 379961300

Current address: Department of Mathematics, University of Colorado, Colorado Springs, Colorado $80933-7150$ 